\title{
Three-dimensional physics-based cellular automata model for granular shear flow
}

\author{
Martin C. Marinack Jr. and C. Fred Higgs III* \\ Mechanical Engineering Department, Carnegie Mellon University, 5000 Forbes Avenue, \\ Pittsburgh, Pennsylvania 15213 USA
}

Granular flows continue to be a complex problem in nature and industry where solid particles exhibit solid, liquid, and gaseous behavior in a manner which is often difficult to predict both locally and globally. In solids processing applications such as pharmaceutical production, food processing, and coal processing, the ability to accurately predict particle flows can improve process efficiency and product effectiveness. The "gold standard" for the modeling and prediction of granular flows is the discrete element method (DEM), which provides a rigorous physical treatment of particle interactions. One possible supplement to DEM-based analysis is lattice-based cellular automata (CA). CA provides a platform for obtaining fast predictions by pairing rule-based mathematics with high-fidelity physics to rapidly model physical processes, such as granular flows. The present work develops a novel three-dimensional (3D) physics-based CA framework for modeling granular shear flows. This 3D CA model extends the framework's modeling capabilities beyond the granular kinetic regime, includes treatments for force chains, and provides reasonably high accuracy in terms of predicting fundamental granular flow experiments. The predictive capabilities and quantitative accuracy of this CA model have been tested and validated against experimental shear flow results from an annular shear cell. Results from this validation work suggest that the model accurately predicts local flow properties. A parametric study varying the particle-particle coefficient of restitution demonstrated the ability of 
the 3D CA model to capture nonlinear trends in the local solid fraction profiles. A time study was also performed to highlight the computational efficiency of the 3D CA framework developed in this work. Results of this study showed that even at relatively low particle counts $\left(10^{4}\right)$, the 3D CA model provides for considerable computational savings, with run times of two orders of magnitude less than a 2D DEM model.

Keywords: Granular materials, Granular flow, Shear flow, Cellular automata, Coefficient of restitution

*higgs@andrew.cmu.edu

\section{INTRODUCTION}

Granular flows have become ubiquitous in both nature and industrial sectors. Examples in nature include avalanches and rockslides, while in industry, granular flows are present in several solids processing applications, such as pharmaceutical production, food processing, and coal transportation and processing. They have even been proposed as a particulate lubricant alternative in extreme environments where traditional oil-based lubricants breakdown $[1,2]$. Within these industrial applications and processes, there are several granular flow phenomena which can cause adverse effects to the cost, yield, or effectiveness of the resultant products or processes themselves. For example, these flow phenomena could include segregation in rotating drums and mixers, jamming in hopper and silo flows, or particle attrition in shear flows. As such, accurate modeling and prediction of granular flows is essential in order to improve industrial processes and designs, as well as to better predict and understand occurrences in nature. Yet, 
granular flows remain difficult to predict and model effectively due to their complex nonlinear and multiphase behavior. This is illustrated by their ability to behave as solids, liquids, or gases, depending on their properties and boundary conditions [3]. Further, the astronomically high number of particles of which granular media are often comprised is another reason they are difficult to model. As a result, the development of faster, more robust, and more accurate granular flow models, and the prediction of an increasingly wider range of flow geometries and systems continues to be an area of extensive study.

Granular flow modeling approaches can be largely divided into two major categories: continuum modeling and discrete modeling. In continuum approaches, granular flow is modeled as a fluid-like continuum, where conservation equations are solved numerically and the choice of constitutive relations becomes essential for model accuracy. As a result, constitutive equations and theories have been developed for a wide range of flow scenarios such as 2D disks in a shear flow [4], flows in the intermediate regime [5, 6], and shear flows of identical highly inelastic spheres [7], just to name a few. Continuum models have also been employed to study a number of applications such as granular lubrication [1, 8-10], and blood flow, where red blood cells are approximated as a granular material [11]. Recent work by Murray et al. [12] has also examined the use of the continuum modeling approach to accurately approximate a continuous particle size distribution.

In discrete modeling approaches, unlike continuum modeling, granular flow is modeled at the single particle (granule) level. Individual particle interactions are modeled, with global flow behavior and predictions coming as the aggregate of these individual particle interactions. The discrete element method (DEM), originally introduced by Cundall and Strack [13], has come to be known as the "gold standard" for discrete granular flow modeling, due to its basis is first- 
principal physics, its rigorous physical treatment of single particle interactions, and its predictive capabilities. DEM has been widely applied in the modeling and prediction of granular flow problems such as classic granular shear flows [14-17], heap formation [18], and flow down inclined planes [19]. Lätzel et al. [20] and McCarthy et al. [21] performed detailed validation studies which compared DEM results against experimental results obtained from lab-scale shear cells. Outside of these more classical granular flow scenarios, the flow of third body particulates as related to material degradation and wear has also been studied using DEM [22-24]. In terms of industrial applications, DEM has been employed extensively inside of the solids processing industry to model and predict granular flow behavior in hoppers [25-27], chutes [28, 29], silos [27, 30], and bladed mixers [31, 32]. While DEM is widely applied due to its high fidelity physics, its main drawback is that it is computationally demanding.

Within the discrete particle modeling community, lattice-based cellular automata (CA) presents itself as a computationally efficient supplement or alternative to a DEM approach. CA, originally introduced by von Neumann [33] in his study of biological systems, provides a modeling platform for obtaining fast first-order predictions of the behavior of many physical systems, such as granular flows. Similar to DEM, CA has been used extensively to study classical granular flow problems and phenomena such as pile formation [34-37], avalanches [37, 38], and granular segregation [34, 38-41]. CA has also been employed in the modeling of solids processing geometries (equipment) like rotating drums (mixers) [41], hoppers [42, 43] and silos $[39,40]$. Previous works by the authors applied a CA approach to examine granular flow lubrication [44], as well as to predict flow inside of an annular shear cell [45, 46]. In the latter works $[45,46]$, detailed experiments were also performed to provide a direct comparison of CA model and experimental results. 
While similar in their wide spread application, CA models and approaches generally lack the rigorous and high-fidelity physics which typifies DEM modeling. Many CA models, including a number of those mentioned previously [34, 38, 42], use a purely rule-based approach for processing particle motion and interactions on the lattice. The rules are generally in the form of look-up tables or equations (rule-based mathematics) and are often based on physical observations of granular flow behavior or basic granular flow physics.

Other CA models [36, 37, 39, 41, 43] go a step further than solely rule-based particle processing through the inclusion of certain experimentally measurable physics-based interaction properties like $e$ and $\mu$. However, these models still do not solve any first-principle physics equations (e.g. conservation of momentum, coefficient of restitution, etc.) during the treatment of particle interactions. Rather, $e$ and $\mu$ are applied in a stochastic manner. For example, Kozicki and Tejchman [43] made use of six probabilistic parameters (p, q, b, k, g, and h), each varying between 0 and 1 , to process granule collisions in their CA model of mass and funnel flow hoppers.

These rule-based and stochastic methods for handling particle motion and interactions often result in models which provide good approximations of the specific systems for which they are designed. However, their lack of physical treatment of behaviors at the particle level becomes limited with regards to the models' predictive capabilities and scope. As a result, previously mentioned works by the authors [44-46] and others [47] have paired CA's computational efficiency with a more physical treatment of particle collision interactions, through the inclusion of physics-based Newtonian particle dynamics. An initial 2D CA model of granular flow lubrication [44] resolved post-collision speeds through solving conservation of momentum and coefficient of restitution $(e)$ equations. However, post-collision directions were still determined 
via look-up tables. Going a step further, the authors expanded the physics included in CA through solving conservation of momentum and $e$ to obtain both the normal speeds and directions of the particles post-collision [45]. Predictions from this 2D CA model [45] were able to provide a good qualitative match to experimental results from an annular shear cell. The quantitative accuracy of the CA model's prediction of the same annular shear cell was improved drastically in the author's next generation of 2D CA model [46]. This model [46] added additional physics into the framework, namely through the inclusion of friction and particle spin effects. Conservation of angular momentum and friction equations were solved to determine the particles post-collision tangential and angular velocities. These elements were ignored during processing in the previous models $[44,45]$. While all three of these models [44-46] made significant strides in moving towards a more physics-based solution of particle collision behavior in CA, there were still a number of limitations in their predictive capabilities and application scope. The major limitations included that the models were only in $2 \mathrm{D}$, they could only be applied to flows in the kinetic or rapid flow regime, and their predictions and accuracy were more qualitative than quantitative. The model presented in this current work attempts to further extend the $\mathrm{CA}$ modeling framework to $3 \mathrm{D}$, while improving upon the aforementioned limitations.

Overall, this work aims to advance the predictive capabilities and broaden the application scope of the CA approach, through the development of a physics-based 3D CA model of granular shear flow. More specifically, this model seeks to increase the physical treatment of particle behavior, while maintaining the computational efficiency and speed that typify a CA approach. The present model creates and divides space into a 3D cubic lattice with an expanded 26 cell neighborhood. The model also introduces physics to handle particle chains which allows 
CA to model conditions outside of purely kinetic flow. These additions all contribute to increases in the accuracy and applicability of the CA model generated in this work.

The results presented in this work include validation of the newly developed 3D CA model against annular shear cell experiments, then simulation results for cylindrical shear cell geometry, and finally parametric studies on particle-particle $e$ and computational run times. Validation studies show that the model provides both qualitative and quantitative accuracy in the prediction of local flow properties. Cylindrical shear cell simulations demonstrate the effects of gravity on the granular shear flow, while the parametric study on particle-particle $e$ exhibits the ability of the model to capture nonlinear flow behavior. Finally, the studies on computational run times demonstrate the ability of the 3D CA model to remain computationally advantageous by comparing CA [45, 46] and DEM [48] frameworks.

\section{VALIDATION EXPERIMENTS: GRANULAR SHEAR CELL DESCRIPTION}

The granular shear cell (GSC), shown in Fig. 1, was used to validate the threedimensional CA model introduced in this work. This same experimental rig was employed for validation of prior CA [45, 46] and DEM [21] models. The GSC is comprised of a stationary outer ring and a quantifiably rough inner wheel. The granular material (monodisperse spherical particles) is placed in the annulus between the rim and wheel, which rotates to produce a Couette granular shear flow. Based on the work of Jenkins and Richman [49], the inner wheel is given a macroscopic roughness by affixing granules along the wheel's edge to create protruding hemispheres. The spacing between neighboring hemispheres quantifies the roughness factor $R$, 
which varies as $0 \leq R \leq 1$. Directly adjacent hemispheres result in a relatively smooth surface defined as $R=0$. Hemispheres spaced exactly one granule diameter apart result in a relatively rough surface defined as $R=1$. The granules being sheared between the inner rotating wheel and outer stationary rim are bounded above and below by a Plexiglas lid and base, respectively. The lid and base create a gap which is slightly larger than one granule diameter, allowing for a single layer of granules to move freely. A more detailed description of this experimental rig and the data acquisition procedure is provided in prior publications [50, 51].

\section{CELLULAR AUTOMATA (CA) MODEL DESCRIPTION}

The new three-dimensional CA modeling approach developed in this work is used to simulate the granular shear cell (GSC) in Fig. 1. This 3D CA framework embodies four basic CA principles. These principles are common to CA modeled systems, such as the 2D CA models previously developed by the authors and utilized in simulating the GSC [45, 46]. Ilachinski [52] outlines these basic CA principles as follows:

(1) Space is represented by a uniform grid made up of discrete cells.

(2) Each cell should be in one of a finite number of fixed states.

(3) Cells may change states only at fixed, regular intervals of time.

(4) States are updated in accordance with fixed local rules operated on an interaction neighborhood. 
Based on these criteria, space is discretized into a three-dimensional cubic lattice (grid), where each grid cell may be empty, occupied by a boundary, or contain a single granule. Each grid space is surrounded by 26 neighboring cells, which comprise the cell's local interaction neighborhood, as shown in Fig. 2. Time is discretized such that the fastest moving particle (or the edge of the inner driving wheel in the case of simulating the GSC) advances exactly 1 grid space during each time step. This discretization scheme guarantees that collision events will not be missed. In classical CA models, the local interaction rules are typically comprised of look-up tables which dictate the manner in which the cells update themselves, or in this case, how granule motion is propagated. However, in this new 3D CA model, the rules consist of solving simple Newtonian-based physics equations inside of the lattice framework. This model uses spherical monodisperse granules.

\section{A. Advantages of the 3D CA model}

With these main CA principles in mind, the advantages and disadvantages of the 3D CA approach developed here, as well as CA modeling of granular flows in general, are examined in detail. The major advantage of using any CA approach is computational efficiency. This computational efficiency is derived from CA's grid-based processing, where global system behavior (e.g. granular flow) is predicted through the modeling of localized interaction behavior (e.g. granule collisions) within rigidly defined interaction neighborhoods. The potential time savings provided by a CA granular flow model (similar to the one developed in this work) was demonstrated in a prior work [48] which developed a 2D multiphase CA-Eulerian framework. In this work, runtimes for the multiphase model using CA to model the particle phase were 
compared directly to runtimes for a multiphase model using the discrete element method (DEM) to model the particle phase. Results showed that with increasing particle counts, CA provided for an increasingly large amount time savings. In fact, even at a relatively low particle count of $10^{4}$, CA displayed reduced run times on the order of 100X (two orders of magnitude) less than DEM run times [48]. Specific studies on the computational efficiency of the 3D CA model produced in the current work, including run times and comparisons to DEM and other CA frameworks, are shown and discussed in the results section.

While computational efficiency is by far the major advantage and reason for using a CA approach in granular flow modeling, there are also some additional, albeit less obvious, advantages to a CA framework. One such advantage is that the CA code can be readily parallelized as a result of its grid-based nature. This parallelization can lead to even more significant computational gains. Another advantage of the specific CA model developed here is that no particle interaction can be missed. This comes as a result of the time discretization scheme, which is based on the fastest moving particle (or boundary) in the system and ensures that no granule moves more than one grid space in a given time step.

\section{B. Disadvantages of the 3D CA model}

The advantages just discussed (section IIIA), namely computational efficiency, serve to illustrate why this CA framework (and CA approaches in general) can be an attractive choice for modeling granular flow systems. However, there are also several disadvantages associated with using a CA approach in granular flow modeling. For instance, because the side length of lattice cells in the current model is set equal to a particle diameter, length scales smaller than a particle 
diameter cannot be resolved within the current framework. The CA approach does not account for particle roughness and its implications on global flow behavior. The current CA model does not give any stress predictions; however, this issue may be addressed in the future model iterations. Additionally, CA frameworks, specifically the 3D CA model developed here, can struggle to model three specific granular flow situations. These situations include polydisperse flows, multi-body contact phenomena, and non-spherical particles. Difficulty in modeling polydisperse flows is related to CA's grid-based nature, where the current model defines the side length of all grid cells to be equivalent to a single particle diameter. Multi-body contact problems arise due to the fact that particle interactions on the CA grid are processed as binary collisions. CA does not include particle stiffness and enduring contacts which can cause difficulty when modeling flows beyond the kinetic regime. Issues with modeling non-spherical particles in CA can be somewhat attributed to the use of a uniformly shaped grid. However, the larger issue with non-spherical modeling resides with difficulty in resolving collision detection and collision processing (updating post-collision velocities). While not impossible, handling these three granular flow issues would require highly complex schemes inside of a CA framework. For instance, polydisperse flows and non-spherical particles could potentially be handled through particle linking or overlapping grids. Contrary to the CA approach developed here, both polydisperse flows and multi-body contact phenomena are handled seamlessly within a basic DEM modeling approach. However, similar to CA, DEM also requires complex schemes for handling non-spherical particles, based on contact detection and collision processing difficulties. Interestingly, the explicit finite element method (FEM), introduced to granular flow modeling by Kabir et al. [53, 54], handles all three of the aforementioned situations seamlessly. However, 
explicit FEM also presents as an extremely computationally demanding approach, far more than CA.

\section{Particle movement on the lattice}

The manner in which the particles move on the $3 \mathrm{D}$ cubic lattice is identical to the manner in which they move on the $2 \mathrm{D}$ rectangular grid, which has been described in detail in previous publications by the authors $[46,48]$. The only difference between the 3D model described in this chapter and the prior $2 \mathrm{D}$ models $[45,46,48]$, is the added ability of the particles to move in the third dimension (z-direction). However, for the sake of completeness, a simple example of particle movement on the 3D lattice is provided in Fig. 3. Before detailing this example, there are a couple of points which should be noted. First, all velocities within the system are normalized by the fastest moving grid cell (the inner rotating wheel in the case of the GSC), which yields velocities between 0.0 and 1.0 "grid cells per time step". Secondly, during any given time step, a granule (particle) must either remain stationary or move one entire grid space. As such, a grid cell can only contain one granule which resides entirely within the cell. Multiple granules cannot be present in a cell, and granules cannot exist across (overlap) multiple cells. The center of a particle coincides with the center of the grid space it occupies, such that the side length of a cubic grid cell is equivalent to a particle diameter. This can be seen in Figs. 2, 3, and 7, which all display spherical particles inside of cubic grid cells.

With these points in mind, consider the granule in Fig. 3(a). This granule possesses a zvelocity $\left(\mathrm{v}_{\mathrm{z}}\right)$ of +0.4 with a $\mathrm{z}$-offset $\left(\mathrm{d}_{\mathrm{z}}\right)$ of 0.0 at an initial time step (time step $\left.=1\right)$. All other velocities $\left(v_{x}\right.$ and $\left.v_{y}\right)$ and offsets $\left(d_{x}\right.$ and $\left.d_{y}\right)$ are assumed 0.0 for this example. These values yield 
an intended motion $\left(\mathrm{s}_{\mathrm{zp}}\right)$ of +0.4 grid spaces (cells) in the z-direction. However, since this value does not exceed the threshold required for a particle to move ( 0.5 grid spaces), the particle remains stationary, continuing to reside in its current grid cell. As a result, Fig. 3(b) shows that the z-offset value is updated to +0.4 , since the particle intended to move +0.4 grid spaces but instead moved 0.0. This offset is then applied during the second time step (time step $=2$ ). As shown in Fig. 3(c), the particle's intended motion during the second time step becomes +0.8 grid spaces, given as the sum of the z-velocity $(+0.4)$ and z-offset $(+0.4)$. Since this value surpasses the 0.5 threshold for movement, the particle now moves one entire grid space in the $+\mathrm{z}$-direction (upward), as witnessed in Fig. 3(d). Finally, because the particle moved +1.0 grid spaces in the zdirection when it only intended to move +0.8 , the particle's z-offset is re-calculated as -0.2 .

\section{Particle-particle interactions}

During any given time step, a granule may attempt to move into any one of its 26 neighbor cells (see Fig. 2). When a particle attempts to move into a cubic grid space already occupied by another particle, the two particles undergo a particle-particle (binary) collision event. During a binary collision, the two particles' pre-collision velocities are first resolved into normal and tangential components. As shown in Fig. 4, for collisions on the 3D grid, this results in one normal component $\left(V_{n}\right)$ and two tangential components $\left(V_{t 1}\right.$ and $\left.V_{t 2}\right)$ for each of the two particles (defined as particle 1 and 2). Similar to a prior 2D model [45], the current model assumes the particles to be smooth and frictionless such that their tangential velocities remain unchanged after a particle-particle collision. The colliding granules' normal velocities are

updated through simultaneously solving the conservation of momentum and coefficient of 
restitution (e) equations displayed as Eqs. (1) and (2), respectively. In Eq. (1), $m_{l}$ and $m_{2}$ define the mass of granule 1 and 2, respectively. Within the current model all granules are assumed to have the same mass. The normal velocities of the colliding granules (1 and 2) before and after the collision are represented as $V_{1, n}^{b}, V_{2, n}^{b}, V_{1, n}^{a}$, and $V_{2, n}^{a}$, respectively. In Eq. (2), the particleparticle coefficient of restitution is given as $e_{p}$. The variables $V_{12, n}^{b}$ and $V_{12, n}^{a}$ respectively define the relative normal velocity $\left(V_{2, n}-V_{1, n}\right)$ between the colliding particles before and after the collision. Solving Eqs. (1) and (2), with the assumption of equal particle masses, yields Eq. (3) to obtain the change in the particles' normal velocities $\left(\Delta V_{1, n}\right.$ and $\left.\Delta V_{2, n}\right)$. Once the changes in normal velocity are obtained (via Eq. (3)), the post-collision velocities of the colliding granules are obtained as shown in Eqs. (4a) and (4b).

$$
\begin{gathered}
m_{1} V_{1, n}^{b}+m_{2} V_{2, n}^{b}=m_{1} V_{1, n}^{a}+m_{2} V_{2, n}^{a} \\
e_{p}=\frac{-V_{12, n}^{a}}{V_{12, n}^{b}}=\frac{-\left(V_{2, n}^{a}-V_{1, n}^{a}\right)}{\left(V_{2, n}^{b}-V_{1, n}^{b}\right)} \\
\Delta V_{1, n}=-\Delta V_{2, n}=\frac{1}{2}\left(1+e_{p}\right) V_{12, n}^{b} \\
V_{1, n}^{a}=V_{1, n}^{b}+\Delta V_{1, n} \\
V_{2, n}^{a}=V_{2, n}^{b}+\Delta V_{2, n}
\end{gathered}
$$

\section{E. Particle-boundary interactions}

When a particle attempts to move into a grid space occupied by a boundary cell, the particle participates in a particle-boundary collision (interaction). The 3D CA framework 
developed in this work is specifically applied to model the GSC (Fig. 1). With this in mind, there are 3 potential particle-boundary interactions which can occur in the model. These include collisions between granules and the Plexiglas base and lid, collisions between granules and the rotating inner wheel, and collisions between granules and the stationary outer rim.

For collisions between a granule and the GSC's base or lid, the granule's post-collision velocities are obtained in the same manner as for particle-particle collisions. Tangential velocity components remain unchanged based on the assumption of smooth (frictionless) surfaces (granule and boundary). The post-collision normal velocity of the colliding particle is obtained through solving the conservation of momentum (Eq. (1)) and $e$ (Eq. (2)) equations, where $m_{2}$ becomes the mass of the boundary. The masses of the base and lid boundaries are assumed to be infinitely larger than the mass of a granule. Additionally, the base and lid boundaries are rigid (maintain zero velocity). With these two facts in mind, solving Eqs. (1) and (2) results in Eq. (5), which obtains the post-collision normal velocity of the granule colliding with the base or lid boundary. In Eq. (5), $e_{b l}$ represents the $e$ between the particle and Plexiglas base or lid boundary.

$$
V_{1, n}^{a}=-e_{b l} V_{1, n}^{b}
$$

With collisions between a granule and the base or lid boundaries now detailed, collisions with the inner (rotating) wheel and outer (stationary) rim remain. Solving for collisions with the outer rim becomes a simplified case of solving for collisions with the inner wheel. As such, only the model's processing of particle collisions with the inner wheel is discussed in detail here. For collisions with the inner wheel or outer rim, the normal component of a particle's post-collision velocity is determined in the exact same way as for collisions with the base or lid, via Eq. (5). 
The only difference is that the $e$ value used in Eq. (5) changes depending on the boundary with which the granule collides. In Eq. (5), $e_{b l}$ is replaced by either $e_{w}$, for particles colliding with the rotating inner wheel, or $e_{o r}$, for particles colliding with the stationary outer rim.

For particles colliding with the driving wheel, resolving the tangential velocity components is based on accounting for the macroscopic roughness (in the form of affixed particles) along the wheel's periphery. Figure 5 illustrates the main difference between collisions in the experimental GSC rig and collisions in the CA model developed here to simulate the GSC. What is shown is that actual collisions inside the experimental rig occur between the particle and physical roughness bumps protruding from the inner wheel. This can be seen in Figs. 5(a) and 5(b) which display a schematic top view and side view of a particle preparing to collide with the rotating inner wheel in the experimental GSC setup. However, these bumps do not exist inside of the grid-based framework of the 3D CA model, which constructs the inner wheel as a rectilinear circle on the cubic lattice. This can be seen in the schematic top and side views displayed in Figs. 5(c) and 5(d). As shown in Fig. 5(b), when a particle collides with the GSC's inner wheel, it can do so in one of three ways. The particle can strike slightly above a protruding hemisphere (case 1), even with a protruding hemisphere (case 2), or slightly below a protruding hemisphere (case 3). This is due to the fact that the spacing between the Plexiglas base and lid in the GSC is $~ 1.6$ times that of a particle diameter, with the protruding roughness bumps centered between the base and lid. Since the roughness hemispheres do not exist physically on the CA grid, the CA model's collision processing must mathematically account for the effect of the roughness bumps on the colliding particle's post-collision tangential velocities.

As such, solving for these tangential velocities is done in a two step process. Whenever a particle attempts to move into a grid cell occupied by the inner wheel boundary, a choice is first 
made at random between one of the three collision cases described above and shown in Fig. 5(b). Then, the granule's post-collision tangential velocities are solved for based on the collision case chosen. For a particle striking slightly above one of the roughness hemispheres (case 1), postcollision tangential velocities are updated via Eqs. (6a) and (6b). Equations (7a) and (7b) update tangential velocities for case 2 (a particle striking even with the roughness hemispheres); while tangential velocities for a particle striking slightly below the roughness hemispheres (case 3) is solved for using Eqs. (8a) and (8b).

$$
V_{1, t 1}^{a}=R\left(0.5 V_{w}\right)+(1-R) V_{1, t 1}^{b}
$$

Case 1:

$$
V_{1, t 2}^{a}=R\left(0.5 V_{w}\right)+(1-R) V_{1, t 2}^{b}
$$

$$
V_{1, t 1}^{a}=R V_{w}+(1-R) V_{1, t 1}^{b}
$$

Case 2:

$$
V_{1, t 2}^{a}=V_{1, t 2}^{b}
$$

$$
V_{1, t 1}^{a}=R\left(0.5 V_{w}\right)+(1-R) V_{1, t 1}^{b}
$$

Case 3:

$$
V_{1, t 2}^{a}=-R\left(0.5 V_{w}\right)+(1-R) V_{1, t 2}^{b}
$$

Each of these 3 equation pairs are based on a linear rule of mixtures, which scales between the impacting particle's initial tangential velocity (in the $t 1$ or $t 2$ direction) and the linear tangential velocity of the inner wheel, $V_{w}$ (in the $t 1$ direction). The relative contribution of these two components is governed by the macroscopic roughness factor, $R$, which serves as the scaling factor and is given by the GSC experimental setup (see section II). This roughness factor, $R$, is experimentally defined as the relative spacing between adjacent hemispheres along the wheel's periphery. 
In an experiment, when $R$ (defined between 0 and 1 ) is large (nearer to 1 ) there is more room between the adjacent hemispheres on the GSC's inner wheel, which results in a larger probability of a particle being struck firmly by a protruding roughness hemisphere. As such, when the CA model processes collisions with high $R$ (for any case), the colliding particle's postcollision tangential velocities have a larger contribution from the wheel's linear tangential velocity $\left(V_{w}\right)$. When $R$ is small (nearer to 0 ) in an experimental GSC trial, there is less room between the adjacent hemispheres on the inner wheel, which results in a larger probability of a particle only being glanced by a protruding roughness hemisphere. Thus, when the CA model processes collisions with low $R$, the colliding particle's post-collision tangential velocities have a larger contribution from the particle's initial tangential velocities and less of a contribution from the wheel's linear tangential velocity $\left(V_{w}\right)$. Finally, for the cases where the particle strikes slightly above or below the roughness hemispheres (1 and 3) a portion of the wheel's tangential velocity is transferred to both tangential directions ( $t 1$ and $t 2)$ based on the angle that would be formed between the granule and roughness hemisphere within the GSC. However for case 2, where the particle strikes even with the roughness hemisphere (in the $t 2$ direction), there is no contribution of the wheel's linear tangential velocity in the $t 2$ direction. As mentioned previously, the processing of particle collisions with the stationary outer rim to obtain postcollision tangential particle velocities is done in an identical manner. The only difference is that the outer rim has zero velocity in all directions, which simplifies the processing equations. 


\section{F. Accounting for the contact region: particle-chain interactions}

The main limitation of prior 2D physics-based CA models of granular shear flow (specifically in the GSC) $[45,46]$ was their inability to simulate any flow outside of the kinetic (rapid) flow regime. While these models [45, 46] provided good predictions for fully kinetic (rapid) granular shear flows, flows containing a contact (quasi-static) regime could not be resolved. Figure 6(a) (adapted from Refs. [46, 51]) displays a transitional flow, consisting of both a kinetic and contact region inside the GSC, for which detailed experimental work has been performed [50, 51]. While DEM was able to seamlessly model this type of transitional flow inside the GSC [21], prior 2D physics-based CA models could not resolve or predict this flow type due to the presence of the contact region. As shown in Fig. 6(b), when attempting to model such a flow, the previously developed frameworks $[45,46]$ would produce a total stagnation of the flow. Once at steady-state, all of the granules within the simulated GSC geometry would come to rest, away from the inner rotating wheel (shearing surface). This stagnation results from the fact that in a GSC experiment, such as the one shown in Fig. 6(a), granules in the contact regime are tightly packed and form networks of force chains and enduring contact forces. This means that a granule colliding with the contact region would almost instantaneously rebound from the contact region and move back towards the inner wheel. However, the sequential and grid-based processing nature of CA handles momentum transfer along the contact regime's force chains as a series of individual instantaneous collision events, which occur over numerous time steps. This leads to the rapid dissipation of an impacting particle's initial velocity and results in its eventual stagnation. As this continues to occur over time, eventually all the particles cluster away from the driving wheel. 
In order to resolve the issue just discussed and expand the physics-based CA approach beyond purely kinetic flows, the 3D physics-based CA framework developed in this work provides for a treatment of particle-chain interactions. However, it should be noted that the current particle-chain treatment is in place to account for the fact that CA does not inherently include particle stiffness or account for multiple and enduring contact. Within the current model, whenever the particle being processed encounters another particle on the grid, it first checks to see if that particle is part of a chain of particles extending to one of the boundaries. If so, the particle being processed updates its normal velocity according to Eq. (9). Otherwise, it performs a typical particle-particle collision as detailed previously (see section IIID). It should be stressed that the chain must be direct (in the direction of the impacting particle's motion) and complete (unbroken) for a particle-chain interaction to take place. Figure 7 demonstrates a situation in which a particle-chain interaction would occur, thereby making use of Eq. (9) to update the impacting particle's normal velocity. In Fig. 7, the blue particle on the far left is preparing to impact the unbroken chain of red particles extending to the green boundary cell on the far right. When a particle-chain interaction occurs, the impacting particle's momentum is transferred through the particles along the chain to the wall, and then back through the chain particles. As such, Eq. (9) is derived as the combination of a series of particle-particle collisions (Eq. (3)) and a single particle-boundary collision (Eq. (5)). The series of particle-particle collisions (Eq. (3)) is scaled by the number of particles in the chain $(N)$, similar to what is shown in Meriam and Kraige [55]. Currently Eq. (9) is shown for a chain extending to the outer rim (as it makes use of $e_{o r}$ ) however, it can be applied to any boundary by simply changing $e_{o r}$ to the appropriate $e$ value. 


$$
\Delta V_{1, n}=-V_{1, n}^{b}\left\{\left[e_{o r}\left(\left(\frac{1}{2}\left(1+e_{p}\right)\right)^{2 N}\right)\right]+1\right\}
$$

Revisiting Fig. 7, if the particle-chain collision treatment in Eq. (9) were not being employed, the blue particle at the far left would strike the chain of red particles and set off a series of particle-particle collisions (and a particle-boundary collision) that would be processed individually (using Eqs. (3) and (5)) over numerous time steps. Due to the grid-based and sequential processing nature of $\mathrm{CA}$, over the course of these time steps, additional collisions would also occur along directions not in line with the particle chain. As such, processing would occur in the same manner as described for the previous 2D CA models $[45,46]$ attempting to reproduce simultaneous kinetic and contact regions inside of the GSC (Fig. 5(a)). As with these prior models [45, 46], processing without the use of Eq. (9) in resolving particle-chain collisions would result in stagnation of the blue impacting particle (Fig. 7), and eventually full stagnation of the flow, as in Fig. 6(b). However, by identifying particle chains and employing Eq. (9) to process particle-chain collisions, the current 3D CA model updates the blue particle's velocity (Fig. 7) within a single time step. This prevents stagnation of the impacting (blue) particle in Fig. 7, and in turn, the full flow stagnation problems witnessed in previous models [45, 46]. And so, where these prior models were limited to only simulating entirely kinetic flows, the current 3D physics-based CA approach allows for the modeling of flows containing kinetic (rapid) and contact (quasi-static) regions (transitional flows). 


\section{G. Gravity modeling}

With the extension to 3D, the presently developed CA framework and GSC model need to account for the effects of gravity on the particles. As such, gravity is applied during every time step as a constant change in the particles' z-velocities $\left(\Delta V_{z}\right)$. Based on the CA time discretization scheme, this change is calculated as shown in Eq. (10), where $\Delta t$ and $g$ are the length of a time step and the gravitational acceleration, respectively. Gravity is applied (through the constant $\Delta V_{z}$ in Eq. (10)) to all the granules inside the GSC domain with two exceptions. The first is when a particle is resting on the bottom boundary (Plexiglas base). The second is when a particle is resting on a chain of particles which extends downward to the bottom boundary. In each of these instances, the particle would still feel the force of gravity; however, it would not experience acceleration (increased z-velocity) due to the opposing force from the bottom boundary.

$$
\Delta V_{z}=-\frac{g \Delta t}{V_{w}}
$$

\section{H. Algorithm}

The algorithm for processing a time step within the presently developed 3D CA model is nearly identical to the algorithm used in the authors' previous 2D CA models [45, 46]. This algorithm has been detailed extensively in these prior works [45, 46]. As such, only certain key points are highlighted here. Before a simulation begins, particles are seeded randomly throughout the domain and given small random initial velocities, such that particles will begin to strike the inner (driving) wheel. As particles are processed sequentially, the processing order is randomly 
shuffled at the beginning of each time step. This ensures that no bias is given to the motion of any particle. When a granule is being processed, it either makes a single collision check of the cell it intends to move into or no (zero) collision checks if it remains stationary in its current cell. The computational efficiency of this CA framework derives itself largely from this fact. Finally, the only major difference between the algorithm used in the current 3D CA model and prior models $[45,46]$ is that an extra check is performed when a particle encounters another particle on the grid. In the current model, the additional check is performed to decide whether a particleparticle or particle-chain interaction will take place.

\section{Data Acquisition}

The procedure for data acquisition within the presently develop 3D CA framework is similar to that of prior works $[45,46]$. However, the addition of the third dimension does result in a few noticeable changes in the calculation and obtainment of local flow data. As such, the data acquisition procedure is detailed fully within this section.

For any given simulation, data is only gathered after the model has reached steady-state. This is determined by viewing the total kinetic energy $\left(E_{K}\right)$ of the system vs. time, where $E_{K}$ is obtained via Eq. (11). In Eq. (11), $N$ represents the total number of granules in the GSC domain; while $v_{T, j}, v_{R, j}$, and $v_{z, j}$ respectively define the tangential, radial, and vertical (z) velocity of a given particle $j$. Whenever the $E_{K}$ of the GSC system reaches a constant equilibrium value (the $E_{K}$ vs. time curve flattens out), the system is said to be at steady-state. This will occur whenever the $E_{K}$ imparted to the granules by the rotating inner wheel is balanced by the $E_{K}$ dissipated 
during particle-particle and particle-boundary collisions. The majority of the trials performed in this work simulate 40 real-life seconds of GSC operation in order to ensure that steady-state is achieved. This proves to be more than adequate as most simulations reach steady-state before the 5 second mark.

$$
E_{K}=\sum_{j=1}^{N}\left(\frac{1}{2} m v_{T, j}^{2}+\frac{1}{2} m v_{R, j}^{2}++\frac{1}{2} m v_{z, j}^{2}\right)
$$

After reaching steady-state local flow property data is obtained by averaging over space and time. In terms of time averaging, data is collected and averaged over a minimum of 1,000 time steps. Space averaging is performed by dividing the annular gap between the inner wheel and outer rim of the GSC into six bins. These bin divisions are structured in both the radial and vertical directions, such that local flow properties inside the GSC can be obtained as a function of both radial location (relative distance between the inner wheel and outer rim) and vertical location (relative distance between the base and lid). Radial bin divisions are displayed graphically in Fig. 8(a), which shows a schematic top view of the GSC; while vertical bin divisions are shown in the schematic side view in Fig. 8(b). With the bin divisions in place, local flow property data is averaged across space by obtaining property values of all the particles present within a given bin during a given time step. This data can then be plotted against the normalized radial distance across the annular gap or the normalized vertical distance between the base and lid. The normalized radial distance (bin radius), $r^{*}$, is defined in Eq. (12), where $r_{b i n}, r_{w}$, and $r_{o r}$ define the center radius of the annular bin, the radius of the rotating inner wheel, and the radius of the stationary outer rim. Normalized vertical distance (bin height), $h^{*}$, is given by Eq. 
(13), where $h_{b i n}, h_{b}$, and $h_{l}$ respectively represent the center height of the vertical bin, the height of the base, and the height of the lid. Both $r^{*}$ and $h^{*}$ are also labeled in Fig. 8.

$$
\begin{array}{r}
r^{*}=\frac{\left(r_{b i n}-r_{w}\right)}{\left(r_{o r}-r_{w}\right)} \\
h^{*}=\frac{\left(h_{b i n}-h_{b}\right)}{\left(h_{l}-h_{b}\right)}
\end{array}
$$

There are four local flow properties for which data is obtained and plotted. These four properties include tangential velocity, solid fraction, 2D granular temperature, and 3D granular temperature. In terms of the tangential velocity, the tangential direction is with respect to the inner wheel and outer rim (circular geometry) of the GSC. In the CA simulations, solid fraction is obtained as a solid fraction ratio, which is defined as the ratio of the number of granules present in a given bin to the maximum number of granules which could reside in the bin. This differs from GSC experiments $[50,51]$ where the solid fraction is obtained in $2 \mathrm{D}$ as the ratio of the bin area covered by particles to the total area of the bin. This difference in solid fraction calculation arises from the fact that the CA framework requires a cell to either be empty or entirely occupied by a single particle, meaning that particles cannot pack hexagonally as they can in experiments. Granular temperature is a measure of velocity fluctuations with respect to the mean velocity inside a given bin. Its calculation is derived from the kinetic theory of gases [56]. The current model tracks 2D granular temperature, in addition to 3D granular temperature. This is done for the purpose of comparing with data from GSC experiments, which track particle data in $2 \mathrm{D}$. The average tangential velocity $\left(V_{T, i}\right)$, solid fraction $\left(v_{i}\right)$, and $2 \mathrm{D}\left(T_{2 D, i}\right)$ and $3 \mathrm{D}\left(T_{3 D, i}\right)$ granular temperatures for a given bin $i$ are calculated via Eqs. (14) - (17), respectively. 


$$
\begin{gathered}
V_{T, i}=\frac{1}{N_{i}} \sum_{j=1}^{N_{i}} v_{T, j} \\
v_{i}=\frac{N_{i}}{N_{i, \max }} \\
T_{2 D, i}=\frac{1}{N_{i}} \sum_{j=1}^{N_{i}} \frac{1}{2}\left[\left(v_{T, j}-V_{T, i}\right)^{2}+\left(v_{R, j}-V_{R, i}\right)^{2}\right] \\
T_{3 D, i}=\frac{1}{N_{i}} \sum_{j=1}^{N_{i}} \frac{1}{3}\left[\left(v_{T, j}-V_{T, i}\right)^{2}+\left(v_{R, j}-V_{R, i}\right)^{2}+\left(v_{z, j}-V_{z, i}\right)^{2}\right]
\end{gathered}
$$

In Eq. (14), which calculates the average tangential velocity in bin $i\left(V_{T, i}\right), N_{i}$ and $v_{T, j}$ define the number of particles in bin $i$ and the tangential velocity of particle $j$ residing within bin $i$, respectively. The maximum number of particles that can fit in bin $i$ is represented as $N_{i, m a x}$ in Eq. (15), which solves for the average solid fraction (ratio) of bin i $\left(v_{i}\right)$. In Eq. (16) for the average $2 \mathrm{D}\left(T_{2 D, i}\right)$ granular temperature in bin $i, v_{R, j}$ is the radial velocity of particle $\mathrm{j}$ within the given bin $i$ and $V_{R, i}$ is the average radial velocity in bin $i$. Velocity in the vertical direction must be accounted for in Eq. (17) when calculating the 3D granular temperature in bin $i\left(T_{3 D, i}\right)$. As such, the variables $v_{z, j}$ and $V_{z, i}$ respectively define the vertical (z) velocity of particle $j$ within bin $i$ and the average vertical velocity in bin $i$. It should be mentioned that in Eq. (16), a factor of $1 / 2$ is used since the granular temperature is being calculated in $2 \mathrm{D}$. The factor of $1 / 3$, used in Eq. (17) for obtaining 3D granular temperature, is more commonly witnessed as the granular temperature calculation is based on 3D kinetic theory of gases. 


\section{RESULTS AND DISCUSSION}

The source code for the current 3D CA framework and GSC model was initially developed in Mathematica version 7. The vast majority of the simulations presented here were run on a Dell Optiplex 980 with an Intel (R) Core(TM) i7 processor and 8.00 GB of RAM. In total, four studies were performed in order to both validate and test the capabilities of the 3D CA framework and GSC model. Similar validation and testing was performed on prior 2D DEM [21] and 2D CA [46] models. The studies that are a part of this 3D CA work are detailed in the following sections. They include a comparison of predictions from the presently developed CA model to experimental results from the GSC [51], an examination of shear flow in a cylindrical shear cell geometry (GSC extended in the third dimension), and a parametric study on the

particle-particle coefficient of restitution. Lastly, a study examining the computational efficiency of the current 3D CA model as compared to other CA [45, 46] and DEM [48] models was performed.

\section{A. Experiments vs. modeling: evaluation the 3D CA model}

In order to evaluate the effectiveness of the 3D CA model in predicting the behavior of a granular (shear) flow, predictions from the model were compared to experimental results obtained from the GSC (pictured in Fig. 1). The experimental GSC results used for comparison are taken from previous work [51]. The dimensions for the simulated GSC were set to exactly match those of the experimental rig. All other inputs for the CA simulation and GSC experiment are summarized in Table I. It is important to note that the gap height (distance between the 
Plexiglas base and lid) in the GSC rig is $1.6 d_{g}$ (1.6 granule diameters). However due to the gridbased nature of the CA framework, partial cells are not able to exist. The gap height in the CA model must exist as an integer multiple of the granule diameter $\left(d_{g}\right)$, since this is also the side length of the cubic grid cells. As such, simulations were performed for a gap height of both $1 d_{g}$ ( 1 grid cell) and $2 d_{g}$ ( 2 grid cells) in order to ascertain which dimension resulted in a more accurate prediction of experimental results. While a gap height of $1 d_{g}$ means that granules cannot physically move in the vertical direction, they can still obtain vertical (z) velocities through collisions with the inner wheel and exchange momentum (dissipate energy) through collisions with the base and lid (see section IIIE). Another important point is that the global solid fraction value refers to the solid fraction over the entire annular gap as opposed to Eq. (15), which gives the solid fraction within an individual bin. The coefficient of restitution values $(e)$ were chosen to match those used in previously published CA models $[45,46]$, which simulated identical GSC experiments in terms of the dimensions and materials used (granules and roughness hemispheres). 
TABLE I. Parameter values used in the study comparing 3D CA simulation predictions against GSC experiments.

\begin{tabular}{lcc}
\hline \hline Parameter & Experiment & 3D CA Model \\
\hline Granule material & Steel & \\
Inner wheel material & Steel & \\
Inner wheel rotation rate $(\mathrm{rpm})$ & 240 & 240 \\
Granule diameter $\left(d_{g}, \mathrm{~mm}\right)$ & 4.76 & 4.76 \\
Height of gap $(H)$ & $1.6 d_{g}$ & $1 d_{g}$ and $2 d_{g}$ \\
Roughness factor $(R)$ & 0.6 & 0.6 \\
Global solid fraction $\left(v_{g}\right)$ & 0.71 & 0.71 \\
Particle-particle coefficient of restitution $\left(e_{p}\right)$ & & 0.8 \\
Particle-wheel coefficient of restitution $\left(e_{w}\right)$ & & 0.8 \\
Particle-outer rim coefficient of restitution $\left(e_{o r}\right)$ & & 0.8 \\
Particle-base/lid coefficient of restitution $\left(e_{b l}\right)$ & & 0.8 \\
Number of particles* & & 1282 \\
\hline \hline
\end{tabular}

*Number of particles in CA simulation are adjusted to match the experimental solid fraction.

Figure 9 displays snapshots of the CA-simulated GSC domain at two different times for a gap height of $1 d_{g}$. In Fig. 9(a), the CA simulation of the GSC is shown at start-up ( $\left.t=0\right)$; while Fig. 9(b) shows the simulation once it has reached steady-state $(t>5 \mathrm{~s})$. The dot on the wheel's surface is present only to aid in visualizing rotation. Initially, the granules are randomly seeded (positioned) throughout the GSC domain, as seen in Fig. 9(a). The major thing to notice is that once at steady-state (Fig. 9(b)) the 3D CA simulation predicts and displays the formation of a 
near-wheel kinetic region and a contact region away from the wheel (against the outer rim). This matches well with what is witnessed in the GSC experiments, as shown in Fig. 6(a). Most importantly, the current model does not have the stagnation issues witnessed in the prior 2D models $[45,46]$, where all granules would come to rest at steady-state. It might also be noticed that at steady-state (Fig. 9(b)) the particles in the contact region display a slightly square-like packing instead of packing in a more circular manner as in experiments (Fig. 6(a)). While this effect is small, it can be explained by the fact that circular geometries are being modeled and approximated on a cubic (rectilinear) lattice. However again, the main point to be gained from these snapshots (Fig. 9) is that the 3D CA model properly develops the kinetic and contact regions, and maintains particle flow within the kinetic region.

Predictions of local flow properties from the presently developed 3D CA model are compared to experimental GSC results [51] in Fig. 10 for normalized tangential velocity (Fig. 10 (a)), solid fraction (Fig. 10 (b)), and normalized granular temperature (Fig. 10 (c)). As detailed in section IIIF, predictions from prior 2D CA models of the GSC $[45,46]$ could not be obtained for comparison since these models were only capable of modeling fully kinetic flows. For the flows examined in this work, the presence of the contact region results in a full stagnation of the flow in the prior 2D CA models $[45,46]$, and the inability of these models $[45,46]$ to provide predictions of local flow properties. And so, Fig. 10 compares 3D CA model predictions for both the $1 d_{g}$ and $2 d_{g}$ gap heights against only experimental GSC results (with a gap height of $1.6 d_{g}$ ). The local flow properties shown in Fig. 10 are all plotted versus the normalized bin radius, $r^{*}$ (Eq. (12)), which defines the radial location between the inner wheel and outer rim. As such, $r^{*}$ $=0$ corresponds to the outer surface of the rotating inner wheel, and $r^{*}=1$ corresponds to the inner surface of the stationary outer rim. This range defines the radial boundaries of the annular 
gap. The data points displayed for both the CA simulation predictions and the GSC experiments are obtained as the average of several trials. For the experiments, the data points are given as the average of 5 trials (as detailed in [51]). The data points for the CA model predictions are given as the average of 8 trials, which is the case for all simulation results displayed in this work. Averaging several trials for the CA simulation predictions is done since a small stochastic component is introduced through the particles being given random initial positions and small random initial velocities. In Fig. 10, as well as all the simulation results shown in this work, the horizontal error bars on each data point represent one standard deviation in both the positive and negative direction.

The general experimental trends for the local flow properties (parameters) versus radial position have been fully detailed in prior works $[50,51]$. However, these experimental trends are briefly reviewed here for completeness. Figure 10(a) shows that the tangential velocity is largest near the rotating inner (shearing) wheel $\left(r^{*}=0\right)$ and decreases while moving towards the stationary outer rim $\left(r^{*}=1\right)$. Additionally, the existence of distinct kinetic and contact regions is displayed in Fig. 10(a), with the transition between these regions occurring approximately between $r^{*}=0.4$ and $r^{*}=0.6$. The experimental solid fraction values displayed in Fig. 10(b) are at a minimum near the inner wheel. This occurs as the shearing wheel drives particles out of the inner regions, forming the densely packed contact region in the outermost bins (near the outer rim). In this manner, a trend of steadily increasing solid fraction might be expected when moving from the inner wheel towards the outer rim. However, a maximum solid fraction is witnessed near $r^{*}=0.6$ (near the transition from the kinetic to contact region). This can be explained by the fact that the gap height of $1.6 d_{g}$ inside the GSC allows for particles to overlap slightly in the vertical direction, even though the experimental data is collected and processed in 2D [50,51]. 
With this in mind, particle overlap is most prevalent at the transition between the kinetic and contact regions and leads to larger solid fractions being obtained during experimental processing. It is also noticed in Fig. 10(b) that the predicted solid fraction values from the CA model and the experimentally obtained values from the GSC are plotted on separate horizontal (x) axes. This is due to the fact that the experimental solid fraction can only reach a maximum of $\sim 0.91$, which is the value for $2 \mathrm{D}$ hexagonal packing of circles. In Figure 10(c), the granular temperature displays an identical trend to tangential velocity. The largest granular temperature values occur nearest the inner wheel $\left(r^{*}=0\right)$ and decrease towards the outer rim $\left(r^{*}=1\right)$. This makes sense as the larger velocities in the inner bins (kinetic region) lead to larger velocity fluctuations, and hence larger granular temperature values.

When comparing simulation results from the CA model to the experimental results in Fig. 10, the gap height which provides the better prediction of experimental results must first be determined. In this regard, it is clear from Fig. 10 that using a gap height of $1 d_{g}$ ( 1 grid cell) provided for much better predictions of local flow properties than did a gap height of $2 d_{g}$ ( 2 grid cells). This can be mainly attributed to the fact that using a gap height of $2 d_{g}$ simply results in too much space being available for the particles to move freely without colliding. The reduction in energy dissipating collisions leads to larger particle velocities and velocity fluctuations. This results directly in the model's over-prediction of tangential velocity (Fig. 10(a)) and granular temperature (Fig. 10(c)) when employing a gap height of $2 d_{g}$. The solid fraction prediction by the CA model with $H=2 d_{g}$ predicts lower values across the annular gap than predicted by the model with $H=1 d_{g}$ or obtained from experiment. The fact that a gap height of $2 d_{g}$ allows the particles to fully overlap other particles causes this under-prediction. Since only particles which would be visible from above are counted (in order to mimic experimental processing), fewer 
particles are tallied in each bin and lower solid fractions are predicted. This is in opposition to experiments where partial overlap can cause additional particles to be counted (and higher solid fractions to be obtained) [50,51]. Overall then, it is clear that using a gap height of $1 d_{g}$ ( 1 grid cell) provides for the best prediction of local flow properties (behavior) inside the GSC.

Focusing now on only the predictions given by the CA model with a gap height of $1 d_{g}$, it is seen across all the flow properties in Fig. 10 that the presently developed 3D CA model provides highly accurate quantitative and qualitative predictions of experimental GSC results. In Fig. 10(a), it is seen that the CA model $\left(H=1 d_{g}\right)$ resolves the qualitative trend of kinetic and contact region formation in addition to providing quantitatively accurate results at each tangential velocity data point. Figure 10(b) shows similarly accurate results from the CA simulations with only some slight discrepancies in local solid fraction prediction. These small discrepancies are likely attributable to the slight overlap witnessed during experiments and its previously discussed effect on experimental processing. The 2D granular temperature profiles in Fig. 10(c) show the CA simulation predictions (for $H=1 d_{g}$ ) almost perfectly overlapping the experimental GSC values. Overall then, the comparison results shown in Fig. 10 serve to showcase the presently developed CA model's ability to provide highly qualitatively and quantitatively accurate predictions of shear flow behavior.

\section{B. Cylindrical shear cell study}

In the prior comparison study (section IVA), CA simulations were tailored to model the granular shear cell (GSC) shown in Fig. 1. As such, the gap height $(H)$ between the base and lid of the shear cell was set to only $1 d_{g}$ or $2 d_{g}$, with the $1 d_{g}$ case showing highly accurate 
predictions. In the following study, the 3D capabilities of the model are further explored by simulating a cylindrical shear cell with a gap height of 12 granule diameters $\left(H=12 d_{g}\right)$, which corresponds to 12 cubic grid cells. The input parameters used in this study are displayed in Table II. The inner and outer cylinder radii of the cylindrical shear cell are set equal to the inner wheel and outer rim radii of the GSC. As such, the cylindrical shear cell simulated in this study is essentially a taller GSC. With the much larger gap height, local flow properties are tracked and displayed as a function of both radial $\left(r^{*}\right)$ and vertical position $\left(h^{*}\right)$ inside the annular gap.

TABLE II. CA simulation parameter values for the cylindrical shear cell study.

\begin{tabular}{lc}
\hline \hline Parameter & Value \\
\hline Inner wheel rotation rate $(\mathrm{rpm})$ & 240 \\
Granule diameter $\left(d_{g}, \mathrm{~mm}\right)$ & 4.76 \\
Height of gap $(H)$ & $12 d_{g}$ \\
Roughness factor $(R)$ & 0.6 \\
Global solid fraction $\left(v_{g}\right)$ & 0.38 \\
Particle-particle coefficient of restitution $\left(e_{p}\right)$ & 0.8 \\
Particle-wheel coefficient of restitution $\left(e_{w}\right)$ & 0.8 \\
Particle-outer rim coefficient of restitution $\left(e_{o r}\right)$ & 0.8 \\
Particle-base/lid coefficient of restitution $\left(e_{b l}\right)$ & 0.8 \\
\hline Number of particles & 8304 \\
\hline \hline
\end{tabular}


Figure 11 displays snapshots of a cylindrical shear cell simulation at start-up (Fig. 11(a)) and after the simulation has reached steady-state (Fig. 11(b)). A single granule is fixed on the inner cylinder's upper surface only as a means to visualize rotation. At the start of the simulation, Fig. 11(a) shows that the granules are randomly positioned throughout the annular gap, based on the initial particle seeding process. As a result of gravity's effect over time, the majority of the granules at steady-state (Fig. 11(b)) reside in the lower regions of the annular gap. However due to continuing collisions with the rotating inner wheel, particles still remain and continue to flow in the upper regions of the gap. This comes about as particle-boundary collisions with the inner wheel can drive particles upward (impart a +z-velocity) against the effects of gravity, as detailed in section IIIE.

CA model predictions of local flow properties inside the cylindrical shear cell (Fig. 11) are displayed in Fig. 12. Tangential velocity (Fig. 12(a)), solid fraction (Fig. 12(b)), and 3D granular temperature (Fig. 12(c)) profiles are provided with respect to both radial and vertical position. Radial position (normalized bin radius $r^{*}$ ) and vertical position (normalized bin height $h^{*}$ ) are shown on the left and right vertical (y) axes, respectively. A small representation of the cylindrical shear cell (taken from Fig. 8(b)) is included with each plot (Figs. 12(a) - 12(c)), as a reminder of how $r^{*}$ and $h^{*}$ are defined within the system. In terms of vertical position (bin height), $h^{*}=0$ corresponds to the base of the cylindrical shear cell; while $h^{*}=1$ corresponds to the lid. The data points in between represent average values obtained within the vertical bins shown in Fig. 8(b). Horizontal error bars are present in Fig. 12; however many of them are not visible beyond the data points themselves. Also, note that the solid fraction in Fig. 12(b) is not the volume solid fraction but the ratio of number of granules defined by Eq. (15). 
The radial profiles for the cylindrical shear cell (Fig. 12) show the same trends as those witnessed in the comparison study (Fig. 10) and detailed in the previous section. As such, the analysis given here focuses on the trends in local flow properties as a function of bin height (the vertical profiles). Figure 12(a) shows that the particles have low tangential velocity in the bottom three bins, which then increases rapidly to a maximum velocity in the uppermost (highest) bin $\left(h^{*} \approx 0.9\right)$. This trend can be explained by viewing the vertical profile for solid fraction in Fig. 12(b). The solid fraction profile displays high solid fraction in the lowest three bins $\left(h^{*}<0.5\right)$ due to gravity's effect on the granules. In these lower bins, the high particle counts (solid fraction) result in a large number of particle collisions and energy dissipation, which leads directly to the low tangential velocities shown in Fig. 12(a). Low solid fraction in the upper regions $\left(h^{*}>0.5\right)$ of the annular gap (Fig. 12(b)) allows for granules to move more freely without undergoing energy dissipating collisions. This fact explains the high tangential velocity values displayed in the upper bins (near $h^{*}=1$ ) in Fig. 12(a). In fact, when examining the vertical profiles in Figs. 12(a) and 12(b) it is seen that higher solid fraction values correspond with lower tangential velocities, displaying an inverse relationship between tangential velocity and solid fraction. In Fig. 12(c), the 3D granular temperature shows the same trend as tangential velocity across the height of the cylinder. This makes sense as higher velocities yield higher velocity fluctuations which is the basis for calculating granular temperature (see Eqs. (16) and (17)). 


\section{Local flow properties as a function of particle-particle coefficient of restitution $\left(e_{p}\right)$}

One of the benefits of granular flow modeling is the ability to examine the effects of parameters which cannot be varied in experimental isolation. In this regard, the following section details a parametric study on particle-particle coefficient of restitution $\left(e_{p}\right)$. The effects of $e_{p}$ on local flow properties inside the GSC are studied. These effects cannot be investigated via GSC experiments as varying the granular material in the annular gap would necessitate a change in several parameters beyond simply the $e_{p}$. Table III provides a summary of the input parameter values used in the parametric $e_{p}$ study. The dimensions were set equal to those of the GSC (Fig. 1), and a gap height $(H)$ of $1 d_{g}$ was used as this was shown to give most accurate prediction of GSC behavior (see section IVA). 
TABLE III. CA simulation parameter values for the $e_{p}$ parametric study.

Parameter

Value

Inner wheel rotation rate $(\mathrm{RPM})$

240

Granule diameter $\left(d_{g}, \mathrm{~mm}\right)$

Height of gap $(H)$

$1 d_{g}$

Roughness factor $(R)$

0.6

Global solid fraction $\left(v_{g}\right)$

0.71

Particle-particle coefficient of restitution $\left(e_{p}\right)$

$0.7,0.8,0.9,1.0$

Particle-wheel coefficient of restitution $\left(e_{w}\right)$

0.8

Particle-outer rim coefficient of restitution $\left(e_{o r}\right)$

0.8

Particle-base/lid coefficient of restitution $\left(e_{b l}\right)$

0.8

Number of particles

1282

Figure 13 compares local flow properties versus radial position for the varying $e_{p}$ values shown in Table III. Figure 13(a) shows that tangential velocity values increase with increasing $e_{p}$ values, particularly in the innermost bins (near the inner wheel $\left(r^{*}=0\right)$ ). The velocity profiles in Fig. 13(a) actually begin to converge while moving towards the outer bins (nearer to $r^{*}=1$ ). This trend makes sense since the $e_{p}$ parameter will be more influential on flow properties in sparsely populated and high speed kinetic (rapid) flow regions [46], such as those that exist near the inner rotating wheel $\left(r^{*}=0\right)$. The regions (bins) nearer to the outer stationary outer rim $\left(r^{*}=\right.$ 1) consist primarily of contact (quasi-static) flow, where the particle-particle coefficient of restitution $\left(e_{p}\right)$ is minimally influential on granular flow properties $[26,46]$. The relative 
influence of $e_{p}$ in these different regions can explain the large variation in tangential velocities near the wheel $\left(r^{*}=0\right)$, followed by the convergence of the profiles (Fig. 13(a)) while moving radially outward towards the outer $\operatorname{rim}\left(r^{*}=1\right)$.

The solid fraction profiles in Fig. 13(b) show an interesting trend across the $e_{p}$ values examined. Starting in the near-wheel region (closer to $r^{*}=0$ ), it is seen that smaller $e_{p}$ values correspond to lower solid fractions. This is the case as lower $e_{p}$ results in more particles stagnating in regions away from the wheel as they collide with other particles and experience larger velocity (energy) dissipation than particles with higher $e_{p}$ values. As such, a large number of particles do not make it back to the inner region after being driven away by the rotating inner wheel. On the contrary, higher $e_{p}$ values allow granules to maintain larger velocities after being driven away by the shearing (inner) wheel, which results in the granules being able to return to the near-wheel region more readily. These facts lead directly to the lower near-wheel $\left(r^{*}<0.4\right)$ solid fractions witnessed in Fig. 13(b) for smaller $e_{p}$ values. Additionally, as lower $e_{p}$ values would result in more velocity dissipation and stagnation away from the wheel, it is expected that the solid fraction trend would reverse while moving towards the outer rim $\left(r^{*}=1\right)$. It is indeed shown in Fig. 13(b) that the trend flips between $r^{*}=0.4$ and $r^{*}=0.8$, with lower $e_{p}$ now corresponding to larger solid fraction values. The reversal of the solid fraction trend also makes sense in consideration of the fact that the global solid fraction remains constant and that mass must be conserved. However, in the final bin (data point) nearest to the outer rim $\left(r^{*}=1\right)$, Fig. 13(b) shows that the trend reverses again, displaying some nonlinearity in the results. In this outermost bin the solid fraction shows the same trend as in the near-wheel region where solid fraction decreases with decreasing values of $e_{p}$. This second trend reversal is based on the interplay between the particle-particle $\left(e_{p}\right)$ and particle-outer rim $\left(e_{o r}\right)$ coefficients of restitution. 
For the simulations with $e_{p}=0.9$ and $e_{p}=1.0$, an increase in solid fraction is witnessed in the bin nearest to the stationary outer rim. This increase is most pronounced in the $e_{p}=1.0$ curve which shows a distinct change in concavity. This sharp increase and change in concavity is observed as values of $e_{p}$ are larger than $e_{o r}$, which remains constant at 0.8 across all the simulations. As such, collisions with the outer rim serve as a much larger energy sink than particle collisions, resulting in the stagnation of more particles near the outer rim. At $e_{p}=0.8$ the solid fraction curve shows a smooth and steady increase in solid fraction when moving into the outermost bin. This comes from the fact that particle-particle and particle-outer rim collisions provide equivalent velocity dissipation, as $e_{p}=e_{o r}$. For the $e_{p}=0.7$ profile, the solid fraction actually decreases slightly when moving into the outermost bin. In this instance (where $e_{p}<e_{o r}$ ) particle-particle collisions actually present a larger energy sink than particle collisions with the outer rim. And so, a slightly smaller number of particles coalesce within the bin adjacent to the outer rim. This same nonlinear solid fraction behavior (two trend reversals) was witnessed in the work of Zhou and Khonsari [57] who performed a similar parametric $e_{p}$ study on a granular shear flow using a continuum modeling approach.

Figure 13(c) compares 3D granular temperature profiles for the $e_{p}$ values studied. The 3D granular temperature follows the same general trend as the tangential velocity (Fig. 13(a)) as increasing the value of $e_{p}$ leads to increased 3D granular temperature values. This is similar to the previous two studies (sections IVA and IVB), as once again, increased velocities lead to increased velocity fluctuations and granular temperatures (based on Eq. (17)). Also shown in Fig. 13(c) is that the model predicts significantly higher $3 \mathrm{D}$ granular temperature values for $e_{p}=1.0$ than for the three lower $e_{p}$ values. This can be attributed to two factors. First of all, the tangential velocity values for the $e_{p}=1.0$ profile are also significantly higher than the other $e_{p}$ values. 
Secondly, an $e_{p}$ of 1.0 yields the largest difference between $e_{p}$ and the other $e$ values $\left(e_{w}, e_{o r}\right.$, and $e_{b l}$, which are all set to 0.8 . This means that having an $e_{p}$ of 1.0 provides for the largest variation in velocity dissipation between particle-particle and particle-boundary collisions, thus contributing to increased fluctuations across the annular gap.

In addition to local flow properties, the average steady-state kinetic energy $\left(E_{K}\right)$ as a function of particle-particle coefficient of restitution $\left(e_{p}\right)$ is examined and displayed in Fig. 14. These $E_{K}$ values are obtained by averaging the total kinetic energy of the entire granular shear flow inside the GSC at every time step after the 10 second mark. This ensures that steady-state, which usually occurs before the 5 second mark, has been achieved. As with the local flow parameters, the data points represent the average of eight trials and display vertical error bars representing one standard deviation in either direction. However, the error bars in Fig. 14 are small and not visible beyond the data points themselves. What is shown by Fig. 14 is that the $E_{K}$ of the system increases as $e_{p}$ is increased. This follows from the fact that larger $e_{p}$ values result in less velocity and kinetic energy dissipation during particle-particle collisions. More specifically, $E_{K}$ appears to display a power law increase with respect to $e_{p}$. This makes sense since kinetic energy $\left(E_{K}\right)$ is proportional to velocity squared $\left(V^{2}\right)$, and from Eq. (2) it is shown that the relative post-collision to pre-collision velocity ratio defines (is proportional to) $e_{p}$. In general, these two facts can be used to discern proportionality between $E_{K}$ and $e_{p}^{2}$.

\section{Run time comparison studies}

As mentioned previously, the main benefit or advantage in developing any CA framework or model is the potential time savings it can provide due to its relative computational 
efficiency as compared to other modeling techniques. In this regard, two run time comparison studies are performed to assess the presently developed 3D CA framework's computational efficiency. The first study compares run times from the 3D CA model against previously published models including 2D CA [45], 2D CA with added friction and spin physics [46], and 2D DEM [48]. The second study examines the effects of the initial size of the 3D cubic grid on 3D CA model run times. These studies serve to highlight the potential time savings and ensure that computational efficiency was not overly compromised by the implementation of the third dimension and any additional physics. Input parameters for the code comparison and grid size comparison studies are shown in Table IV. All simulations in this study were run on a Dell Optiplex 980 desktop computer with an Intel(R) Core(TM) i7 processor and 8.00 GB of RAM. It should also be noted that while various high performance computing schemes, namely parallelization, could readily be applied to any of the frameworks used in the run time comparison studies, all of the frameworks are in their simplest form. In other words, no high performance computing schemes, such as parallel computing, have been employed to benefit the run times of any code over any other. In this manner a direct and unbiased comparison of the computational efficiency of the different frameworks can be obtained. 
TABLE IV. CA simulation parameter values for the run time comparison studies.

\begin{tabular}{lcc}
\hline \hline Parameter & Code Comparison & Grid Size \\
& & Comparison \\
\hline Inner wheel rotation rate $(\mathrm{RPM})$ & 260 & 260 \\
Granule diameter $\left(d_{g}, \mathrm{~mm}\right)$ & 1.75 & $1.25,1.75$ \\
Height of gap $(H)$ & $1 d_{g}$ & $12 d_{g}$ \\
Roughness factor $(R)$ & 0.8 & 0.8 \\
Number of granules $(N)$ & $10-10,000$ & $10-10,000$ \\
Particle-particle coefficient of restitution $\left(e_{p}\right)$ & 0.8 & 0.8 \\
Particle-wheel coefficient of restitution $\left(e_{w}\right)$ & 0.8 & 0.8 \\
Particle-outer rim coefficient of restitution $\left(e_{o r}\right)$ & 0.8 & 0.8 \\
Particle-base/lid coefficient of restitution $\left(e_{b l}\right)$ & 0.8 & 0.8 \\
\hline \hline
\end{tabular}

A comparison of the presently developed 3D CA model's run times against other previously published CA [45, 46] and DEM [48] modeling frameworks is provided in Fig. 15. In this code comparison study, normalized run time $\left(t_{R}^{*}\right)$ is plotted against the number of particles being simulated $(N)$. Normalization is done by dividing all the run times by the run time of the fastest model, which was the 2D CA model [45] without any friction or spin considerations. In Fig. 15, the 2D model with friction and spin (2D CA (FS) [46]) shows little to no increase in relative run time as compared to the baseline 2D model [45]. The additional collisions physics associated with adding friction and spin in the 2D CA (FS) model have minimal to no effect on the 2D CA model's run times. 
The presently developed 3D model does show a relative run time increase across the particle counts examined. However, the increase is less than an order of magnitude $(\sim 3 \mathrm{X})$ and remains constant over increasing particle counts $(N)$. Based on what was just discussed for the different 2D models, it is unlikely that this increased run time results from any physics considerations within the particle collisions themselves. Instead, the constant increase in run time can be attributed to the time required to process aspects of the model associated with the presence of the third dimension. For example, the 3D model uses a larger interaction neighborhood (Fig. 2) than in 2D, requires the tracking of an additional velocity and position component for every particle, and generates additional particle-boundary collisions through the presence of the base and lid geometries.

It is also shown in Fig. 15 that all of the CA models display a significant time savings when compared to the 2D DEM framework [48] as particle counts increase. At low particle counts $(N \leq 100)$, the relative $2 \mathrm{D}$ DEM run times are actually lower than the $3 \mathrm{D}$ CA model and nearly equivalent to those of the 2D CA models [45, 46]. This can be attributed to the fact that the DEM framework is in 2D, and that at low $N$, the effects of collision searching in DEM are minimized. However, as particle counts increase to $N=1000$ and beyond, Fig. 15 shows that the disparity between the relative run times of the 2D DEM and CA frameworks becomes increasingly large. In fact, at $N=10,000$, the presently developed 3D CA framework shows a large computational savings of two orders of magnitude when compared to the 2D DEM framework [48]. As detailed in prior work [48], certain optimizations [58, 59] could be applied to the current $O\left(N^{2}\right)$ collision detection scheme used in the DEM code to reduce DEM run times. Despite this fact, it is still maintained that the CA frameworks $(O(N)$ collision detection), including the $3 \mathrm{D}$ model, would provide significant computational savings due to the fact that 
certain speedups, such as parallelization, could be applied to both the DEM and CA frameworks. In this manner, CA would maintain its computational advantage [48].

With any CA approach, one of the common concerns with computational efficiency is the effect of the grid size used to simulate the domain being studied. To this end, Fig. 16 shows a comparison between 3D CA simulations of the GSC with different grid sizes (a different number of grid cells). The number of cells was varied by changing the particle diameter, which defines the side length of the cubic grid cells. Figure 16 shows that nearly doubling the amount of grid cells does cause an increase in the run time of the model. Yet, the increase is very slight and remains constant as the number of particles in the domain $(N)$ increases. This constant increase makes sense since the additional cells require more memory to be used, regardless of the number of particles present in the domain. The relatively small size of the increase also makes sense as the number of particle collisions should remain roughly the same at each particle count, and since the relative increase in grid size is fairly small.

\section{CONCLUSION}

In this work, a three-dimensional (3D) physics-based CA framework for modeling granular flows is developed and applied to model flow inside of an annular shear cell. This model improves upon prior 2D models $[45,46]$ in both predictive capabilities and accuracy. Additional physics for particle chain treatments allows for CA to model flows outside of purely kinetic regimes. The capacity of the current model to accurately predict shear flow properties was demonstrated by comparing CA simulation predictions to experimental results. In this comparison (validation) study, the 3D CA model provided highly accurate quantitative and 
qualitative predictions of experimental GSC behavior [51], namely local flow property profiles. Simulations of a cylindrical shear cell, similar to a taller GSC, highlighted the full 3D capabilities of the presently developed 3D CA framework, such as the ability to track local flow properties as a function vertical position. A parametric study on particle-particle coefficient of restitution $\left(e_{p}\right)$ demonstrated increased tangential velocity, granular temperature, and kinetic energy for larger values of $e_{p}$. However, the most interesting find in the $e_{p}$ parametric study was that the model was able to capture the highly nonlinear solid fraction behavior, where a concavity switch occurs when the $e_{p}$ exceeds the $e_{o r}$; this was previously documented in continuum modeling work [57]. The applicability and accuracy of CA for increasingly nonkinetic, intermediate, and even quasi-static regimes is something which would require further development, testing and validation; however, the current model provides an initial approach and physics-based framework for handling a flow which is not purely kinetic. The potential time savings provided by the current 3D CA model was exhibited through a comparison of run times with 2D CA models [45, 46] and a DEM framework [48]. The 3D CA model showed a small, but constant increase in run times as compared to the 2D CA models. However, a large computational advantage was still maintained when comparing to the 2D DEM model. At a particle count of $10^{4}$, the 3D CA model showed run times which were two orders of magnitude faster than the 2D DEM model. This pairing of computational efficiency with predictive accuracy in the current model demonstrates the potential for the general CA approach to produce accurate high-speed models of granular flow systems. This point is particularly important as granular flow modeling continues to push towards industrial granular flow applications with vastly increased particle counts. Future work with the 3D CA framework will involve extending the framework to model additional granular flow situations. 


\section{ACKNOWLEDGMENTS}

This material is based upon work supported by the National Science Foundation Graduate Research Fellowship under Grant No. 0946825. The experimental segment of this work was partially supported by the NETL/DOE.

[1] C.F. Higgs, J. Tichy, Granular Flow Lubrication: Continuum Modeling of Shear Behavior, Journal of Tribology 126 (2004) 499-510.

[2] C.-M. Yu, K. Craig, J. Tichy, Granular collision lubrication, Journal of Rheology 38 (1994) 921-36.

[3] H.M. Jaeger, S. Nagel, R., R.P. Behringer, Granular solids, liquids, and gases, Reviews of Modern Physics 68 (1996) 1259-73.

[4] H.H. Shen, N.L. Ackermann, Constitutive Equations for a Simple Shear Flow of a Disk Shaped Granular Mixture, International Journal of Engineering Science 22 (1984) 829-43.

[5] P.C. Johnson, R. Jackson, Frictional-Collisional Constitutive Relations for Granular Materials, with Application to Plane Shearing, Journal of Fluid Mechanics 176 (1987) 67-93.

[6] G. Tardos, S. McNamara, I. Talu, Slow and intermediate flow of a frictional bulk powder in the Couette geometry, Powder Technology 131 (2003) 23-39.

[7] C.-S. Chou, M.W. Richman, Constitutive Theory for Homogeneous Granular Shear Flows of Highly Inelastic Spheres, Physica A 259 (1998) 430-48.

[8] W.G. Sawyer, J.A. Tichy, Lubrication With Granular Flow: Continuum Theory, Particle Simulations, Comparison With Experiment, Journal of Tribology 123 (2001) 777.

[9] Y.-R. Jeng, H.-J. Tsai, Grain Flow for Rough Surfaces Considering Grain/Grain Collision Elasticity, Journal of Tribology 127 (2005) 837.

[10] J. Tichy, Y. Berthier, I. Iordanoff, A Continuum Description of Dense Granular Lubrication Flow, Journal of Tribology 130 (2008) 031301.

[11] M. Massoudi, J. Kim, J.F. Antaki, Modeling and numerical simulation of blood flow using the theory of interacting continua, International Journal of Non-Linear Mechanics 47 (2012) 506-20.

[12] J.A. Murray, S. Benyahia, P. Metzger, C.M. Hrenya, Continuum representation of a continuous size distribution of particles engaged in rapid granular flow, Physics of Fluids 24 (2012) 083303.

[13] P.A. Cundall, O.D.L. Strack, Discrete Numerical Model for Granular Assemblies, Geotechnique 29 (1979) 47-65.

[14] S.R. Dahl, R. Clelland, C.M. Hrenya, The effects of continuous size distributions on the rapid flow of inelastic particles, Physics of Fluids 14 (2002) 1972.

[15] S.R. Dahl, R. Clelland, C.M. Hrenya, Three-dimensional, rapid shear flow of particles with continuous size distributions, Powder Technology 138 (2003) 7-12.

[16] W. Ketterhagen, J. Curtis, C. Wassgren, Stress results from two-dimensional granular shear flow simulations using various collision models, Physical Review E 71 (2005) 061307. 
[17] I. Iordanoff, K. Elkholy, M.M. Khonsari, Effect of particle size dispersion on granular lubrication regimes, Proceedings of the Institution of Mechanical Engineers, Part J: Journal of Engineering Tribology 222 (2008) 725-39.

[18] H.G. Matuttis, S. Luding, H.J. Herrmann, Discrete element simulations of dense packings and heaps made of spherical and non-spherical particles, Powder Technology 109 (2000) 278-92.

[19] X.M. Zheng, J.M. Hill, Molecular dynamics simulation of granular flows: Slip along rough inclined planes, Computational Mechanics 22 (1998) 160-6.

[20] M. Latzel, S. Luding, H.J. Herrmann, D.W. Howell, R.P. Behringer, Comparing simulation and experiment of a 2D granular Couette shear device, The European Physical Journal E - Soft Matter 11 (2003) 325-33.

[21] J.J. McCarthy, V. Jasti, M. Marinack, C.F. Higgs, Quantitative validation of the discrete element method using an annular shear cell, Powder Technology 203 (2010) 70-7.

[22] N. Fillot, I. Iordanoff, Y. Berthier, A Granular Dynamic Model for the Degradation of Material, Journal of Tribology 126 (2004) 606.

[23] N. Fillot, I. Iordanoff, Y. Berthier, Simulation of Wear Through Mass Balance in a Dry Contact, Journal of Tribology 127 (2005) 230.

[24] I. Iordanoff, N. Fillot, Y. Berthier, Numerical study of a thin layer of cohesive particles under plane shearing, Powder Technology 159 (2005) 46-54.

[25] W. Ketterhagen, J. Curtis, C. Wassgren, A. Kong, P. Narayan, B. Hancock, Granular segregation in discharging cylindrical hoppers: A discrete element and experimental study, Chemical Engineering Science 62 (2007) 6423-39.

[26] A. Anand, J. Curtis, C. Wassgren, B. Hancock, W. Ketterhagen, Predicting discharge dynamics from a rectangular hopper using the discrete element method (DEM), Chemical Engineering Science 63 (2008) 5821-30.

[27] T. Goda, F. Ebert, Three-dimensional discrete element simulations in hoppers and silos, Powder Technology 158 (2005) 58-68.

[28] H. Li, G.R. McDowell, I.S. Lowndes, A laboratory investigation and discrete element modeling of rock flow in a chute, Powder Technology 229 (2012) 199-205.

[29] Y. Fan, K.M. Hill, Phase Transitions in Shear-Induced Segregation of Granular Materials, Physical Review Letters 106 (2011) 218301.

[30] V. Vidyapati, S. Subramaniam, Granular Flow in Silo Discharge: Discrete Element Method Simulations and Model Assessment, Industrial and Engineering Chemisty Research 52 (2013) 13171-82.

[31] B. Remy, T.M. Canty, J.G. Khinast, B.J. Glasser, Experiments and simulations of cohesionless particles with varying roughness in a bladed mixer, Chemical Engineering Science 65 (2010) 4557-71.

[32] S.L. Conway, A. Lekhal, J.G. Khinast, B.J. Glasser, Granular flow and segregation in a four-bladed mixer, Chemical Engineering Science 60 (2005) 7091-107.

[33] J. Von Neumann, ed. Theory of Self-Reproducing Automata. Urbana and London: University of Illinois Press 1966.

[34] A.D. Fitt, P. Wilmott, Cellular-automaton model for segregation of a two-species granular flow, Physical Review A 45 (1992) 2383.

[35] E. Goles, Sand pile automata, Annales de I'Institut Henri Poincare Physique Theorique 56 (1992) 75-90.

[36] J.J. Alonso, H.J. Herrmann, Shape of the Tail of a Two-Dimensional Sandpile, Physical Review Letters 76 (1996) 4911-4.

[37] A. Karolyi, J. Kertesz, Lattice-gas model of avalanches in a granular pile, Physical Review E 57 (1998) 852-6.

[38] B. Marks, I. Einav, A cellular automaton for segregation during granular avalanches, Granular Matter 13 (2011) 211-4. 
[39] A. Karolyi, J. Kertesz, S. Havlin, H.A. Makse, H.E. Stanley, Filling a silo with a mixture of grains: friction-induced segregation, Europhysics Letters 44 (1998) 386-92.

[40] P. Cizeau, H.A. Makse, H.E. Stanley, Mechanisms of granular spontaneous stratification and segregation in two-dimensional silos, Physical Review E 59 (1999) 4408-21.

[41] A.C. Santomaso, R. Artoni, P. Canu, Controlling axial segregation in drum mixers through wall friction: Cellular automata simulations and experiments, Chemical Engineering Science 90 (2013) 15160.

[42] W.G. Baxter, R.P. Behringer, Cellular automata models of granular flow, Physical Review A 42 (1990) 1017-20.

[43] J. Kozicki, J. Tejchman, Application of a cellular automaton to simulations of granular flow in silos, Granular Matter 7 (2005) 45-54.

[44] V.K. Jasti, C.F. Higgs, A Lattice-Based Cellular Automata Modeling Approach for Granular Flow Lubrication, Journal of Tribology 128 (2006) 358.

[45] V.K. Jasti, C.F. Higgs, A fast first order model of a rough annular shear cell using cellular automata, Granular Matter 12 (2010) 97-106.

[46] M.C. Marinack Jr., C.F. Higgs III, The Inclusion of Friction in Lattice-Based Cellular Automata Modeling of Granular Flows, Journal of Tribology 133 (2011) 031302.

[47] W. Österle, A.I. Dmitriev, H. Kloß, Possible impacts of third body nanostructure on friction performance during dry sliding determined by computer simulation based on the method of movable cellular automata, Tribology International 48 (2011) 128-36.

[48] M.C. Marinack Jr., J.N. Mpagazehe, C.F. Higgs III, An Eulerian, Lattice-based Cellular Automata Approach for Modeling Multiphase Flows, Powder Technology 221 (2012) 47-56.

[49] J.T. Jenkins, M.W. Richman, Boundary Conditions for Plane Flows of Smooth, Nearly Elastic, Circular Disks, Journal of Fluid Mechanics 171 (1986) 53-69.

[50] V. Jasti, C. Higgs, Experimental study of granular flows in a rough annular shear cell, Physical Review E 78 (2008) 041306.

[51] M.C. Marinack Jr., V.K. Jasti, Y.E. Choi, C.F. Higgs, Couette grain flow experiments: The effects of the coefficient of restitution, global solid fraction, and materials, Powder Technology 211 (2011) 144-55.

[52] A. Ilachinski, Cellular Automata A Discrete Universe, World Scientific Publishing Co. Pte. Ltd., Singapore, 2001.

[53] M.A. Kabir, M.R. Lovell, C.F. Higgs, Utilizing the Explicit Finite Element Method for Studying Granular Flows, Tribology Letters 29 (2008) 85-94.

[54] M.A. Kabir, V.K. Jasti, C.F. Higgs, M.R. Lovell, An evaluation of the explicit finite-element method approach for modelling dense flows of discrete grains in a Couette shear cell, Proceedings of the Institution of Mechanical Engineers, Part J: Journal of Engineering Tribology 222 (2008) 715-23.

[55] J.L. Meriam, L.G. Kraige, Impact, in: L. Wojcik (Ed.) Engineering Mechanics: Dynamics, 6th Edition, John Wiley \& Sons, Inc., Hoboken, NJ, 2007, pp. 221-33.

[56] C.K.K. Lun, S.B. Savage, D.J. Jeffrey, N. Chepurniy, Kinetic Theories for Granular Flow: Inelastic Particles in Couette Flow and Slightly Inelastic Particles in a General Flowfield, Journal of Fluid Mechanics 140 (1984) 223-56.

[57] L. Zhou, M.M. Khonsari, Flow Characteristics of a Powder Lubricant Sheared Between Parallel Plates, Journal of Tribology 122 (2000) 147-54.

[58] L. Verlet, Computer 'experiments' on classical fluids. I. thermodynamical properties of LennardJones molecules, Physical Review 159 (1967) 98-103.

[59] Z. Yao, J.-S. Wang, M. Cheng, Improved $\mathrm{O}(\mathrm{N})$ neighbor list method using domain decomposition and data sorting in: NSTI Nanotechnology Conference and Trade Show, Boston, Massachusetts, 2004. 


\section{FIGURE CAPTIONS}

FIG. 1. (Color online) Top view of the granular shear cell (GSC); gravity acts into the page.

FIG. 2. (Color online) Three-Dimensional granule (particle) interaction neighborhood. A particle's interaction neighborhood consists of three planes, which yield a total of 26 neighbor cells. The planes above and below the particle contain 9 neighbor cells each. The plane in which the particle resides contains the remaining 8 neighbor cells, and is analogous to the interaction neighborhood witnessed on a two-dimensional (square) lattice.

FIG. 3. (Color online) An example of how granules move on the 3D CA (cubic) lattice. (a) At an initial time step the granule possesses a z-velocity of +0.4 "grid spaces per time step" and a $\mathrm{z}$ offset of 0.0 , which combine to give an intended motion of +0.4 grid cells. (b) Since the granule's intended movement is less than 0.5 , the particle remains stationary and only updates its offset value to +0.4 . (c) At time step $=2$, the updated offset and velocity are summed to yield an intended motion of +0.8 grid spaces in the z-direction. (d) With the intended motion exceeding 0.5, the granule moves +1.0 cells in the $+\mathrm{z}$-direction and updates its offset to -0.2 .

FIG. 4. (Color online) Two particles preparing to undergo a binary collision. 
FIG. 5. (Color online) Schematic views of a particle preparing to participate in a collision with the rotating inner wheel: (a) Top view representation for the experimental GSC rig. (b) Side view representation for the experimental GSC rig. (c) Top view for the CA model of the GSC. (d) Side view for the CA model of the GSC.

FIG. 6. (Color online) Modeling granular shear flows outside of the kinetic regime: (a) GSC experiment producing a transitional flow (containing both a kinetic and contact region) (b) Typical simulation result from prior models when attempting to reproduce this flow. All of the granules stagnate away from the rotating inner wheel as steady-state is reached.

FIG. 7. (Color online) A particle preparing to participate in a particle-chain collision interaction. The particle on the far left will strike the direct and unbroken chain of particles extending to the boundary cell on the far right

FIG. 8. (Color online) Division of the annular gap of the simulated GSC, a concentric cylinder geometry, into (a) radial bins and (b) vertical bins for data acquisition.

FIG. 9. (Color online) Snapshots of the CA simulation of the GSC at (a) start-up and at (b) steady-state for a gap height of $1 d_{g}$.

FIG. 10. (Color online) Local flow properties vs. radial location, comparing the CA model of the GSC against experimental GSC results: (a) normalized tangential velocity, (b) solid fraction, and (c) normalized 2D granular temperature. 
FIG. 11. (Color online) Snapshots of the CA simulation of a cylindrical shear cell $\left(H=12 d_{g}\right)$ at (a) start-up and at (b) steady-state.

FIG. 12. (Color online) Local flow properties vs. radial and vertical location in a cylindrical shear cell: (a) normalized tangential velocity, (b) solid fraction, and (c) normalized 3D granular temperature.

FIG. 13. (Color online) Local flow properties vs. radial location as a function of particle-particle coefficient of restitution $\left(e_{p}\right)$ in the GSC: (a) normalized tangential velocity, (b) solid fraction, and (c) normalized 3D granular temperature.

FIG. 14. Average steady-state kinetic energy $(t>10 \mathrm{~s})$ vs. particle-particle coefficient of restitution $\left(e_{p}\right)$ in the GSC.

FIG. 15. (Color online) Normalized run time $\left(t_{R}^{*}=t_{R} / t_{R(2 D C A)}\right)$ vs. number of particles simulated, comparing the computational efficiency of various CA models.

FIG. 16. (Color online) Run time vs. number of particles simulated, comparing 3D CA simulations with different grid sizes (number of cubic grid cells). 
TABLE I. Parameter values used in the study comparing 3D CA simulation predictions against GSC experiments.

\begin{tabular}{lll}
\hline \hline Parameter & Experiment & 3D CA Model \\
\hline Granule material & Steel & \\
Inner wheel material & Steel & \\
Inner wheel rotation rate $(\mathrm{rpm})$ & 240 & 240 \\
Granule diameter $\left(d_{g}, \mathrm{~mm}\right)$ & 4.76 & 4.76 \\
Height of gap $(H)$ & $1.6 d_{g}$ & $1 d_{g}$ and $2 d_{g}$ \\
Roughness factor $(R)$ & 0.6 & 0.6 \\
Global solid fraction $\left(v_{g}\right)$ & 0.71 & 0.71 \\
Particle-particle coefficient of restitution $\left(e_{p}\right)$ & & 0.8 \\
Particle-wheel coefficient of restitution $\left(e_{w}\right)$ & & 0.8 \\
Particle-outer rim coefficient of restitution $\left(e_{o r}\right)$ & & 0.8 \\
Particle-base/lid coefficient of restitution $\left(e_{b l}\right)$ & 1633 & 0.8 \\
Number of particles* & & 1282 \\
\hline \hline Number & & \\
\hline
\end{tabular}

*Number of particles in CA simulation are adjusted to match the experimental solid fraction. 
TABLE II. CA simulation parameter values for the cylindrical shear cell study.

\begin{tabular}{ll}
\hline \hline Parameter & Value \\
\hline Inner wheel rotation rate $(\mathrm{rpm})$ & 240 \\
Granule diameter $\left(d_{g}, \mathrm{~mm}\right)$ & 4.76 \\
Height of gap $(H)$ & $12 d_{g}$ \\
Roughness factor $(R)$ & 0.6 \\
Global solid fraction $\left(v_{g}\right)$ & 0.38 \\
Particle-particle coefficient of restitution $\left(e_{p}\right)$ & 0.8 \\
Particle-wheel coefficient of restitution $\left(e_{w}\right)$ & 0.8 \\
Particle-outer rim coefficient of restitution $\left(e_{o r}\right)$ & 0.8 \\
Particle-base/lid coefficient of restitution $\left(e_{b l}\right)$ & 0.8 \\
Number of particles & 8304 \\
\hline \hline
\end{tabular}


TABLE III. CA simulation parameter values for the $e_{p}$ parametric study.

\begin{tabular}{ll}
\hline \hline Parameter & Value \\
\hline Inner wheel rotation rate (RPM) & 240 \\
Granule diameter $\left(d_{g}, \mathrm{~mm}\right)$ & 4.76 \\
Height of gap $(H)$ & $1 d_{g}$ \\
Roughness factor $(R)$ & 0.6 \\
Global solid fraction $\left(v_{g}\right)$ & 0.71 \\
Particle-particle coefficient of restitution $\left(e_{p}\right)$ & $0.7,0.8,0.9,1.0$ \\
Particle-wheel coefficient of restitution $\left(e_{w}\right)$ & 0.8 \\
Particle-outer rim coefficient of restitution $\left(e_{o r}\right)$ & 0.8 \\
Particle-base/lid coefficient of restitution $\left(e_{b l}\right)$ & 0.8 \\
Number of particles & 1282 \\
\hline \hline
\end{tabular}


TABLE IV. CA simulation parameter values for the run time comparison studies.

\begin{tabular}{lll}
\hline \hline Parameter & Code Comparison & Grid Size Comparison \\
\hline Inner wheel rotation rate (RPM) & 260 & 260 \\
Granule diameter $\left(d_{g}, \mathrm{~mm}\right)$ & 1.75 & $1.25,1.75$ \\
Height of gap $(H)$ & $1 d_{g}$ & $12 d_{g}$ \\
Roughness factor $(R)$ & 0.8 & 0.8 \\
Number of granules $(N)$ & $10-10,000$ & $10-10,000$ \\
Particle-particle coefficient of restitution $\left(e_{p}\right)$ & 0.8 & 0.8 \\
Particle-wheel coefficient of restitution $\left(e_{w}\right)$ & 0.8 & 0.8 \\
Particle-outer rim coefficient of restitution $\left(e_{o r}\right)$ & 0.8 & 0.8 \\
Particle-base/lid coefficient of restitution $\left(e_{b l}\right)$ & 0.8 & 0.8 \\
\hline \hline
\end{tabular}


Figure_1
Click here to download high resolution image

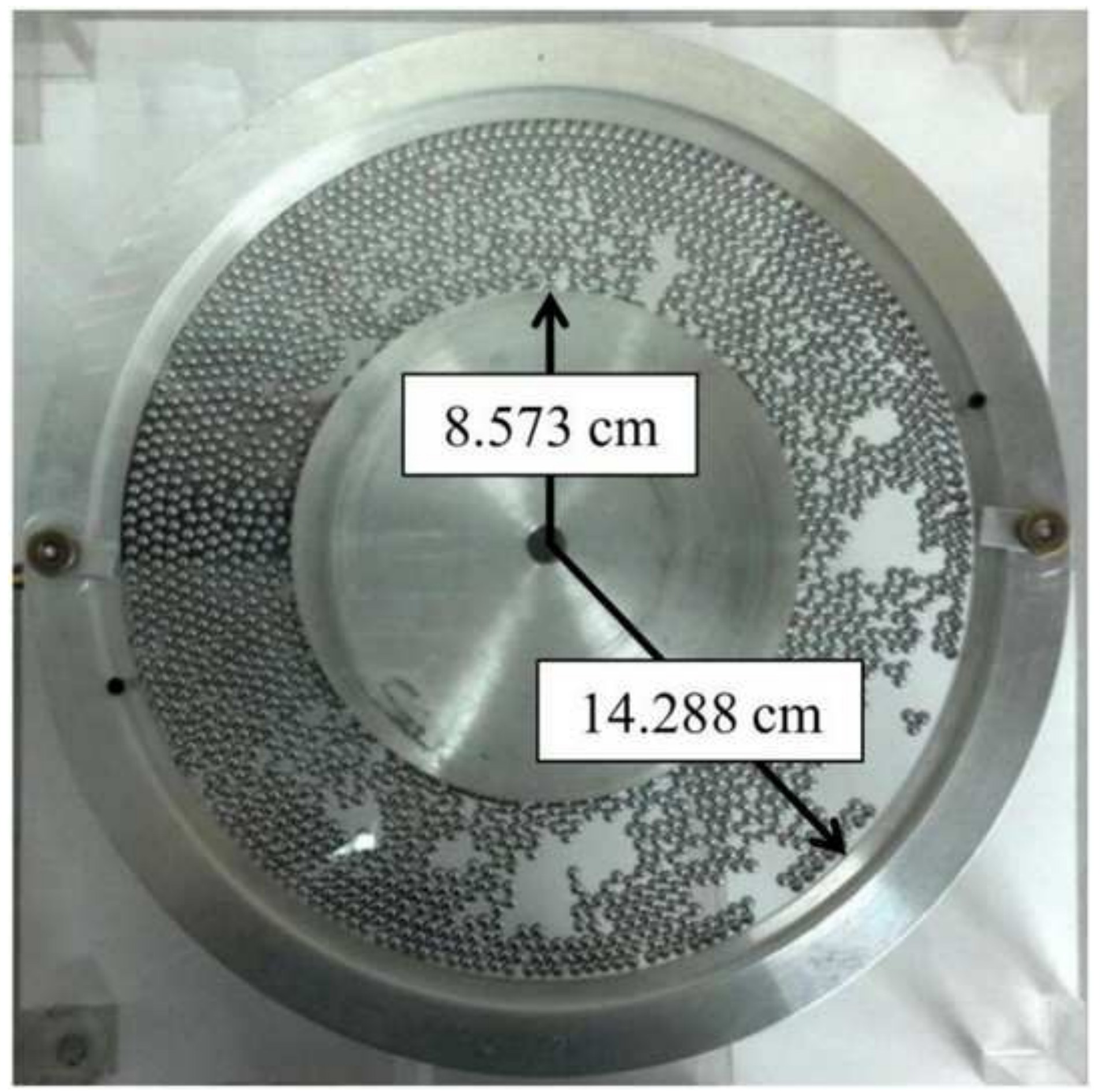

Figure_1 


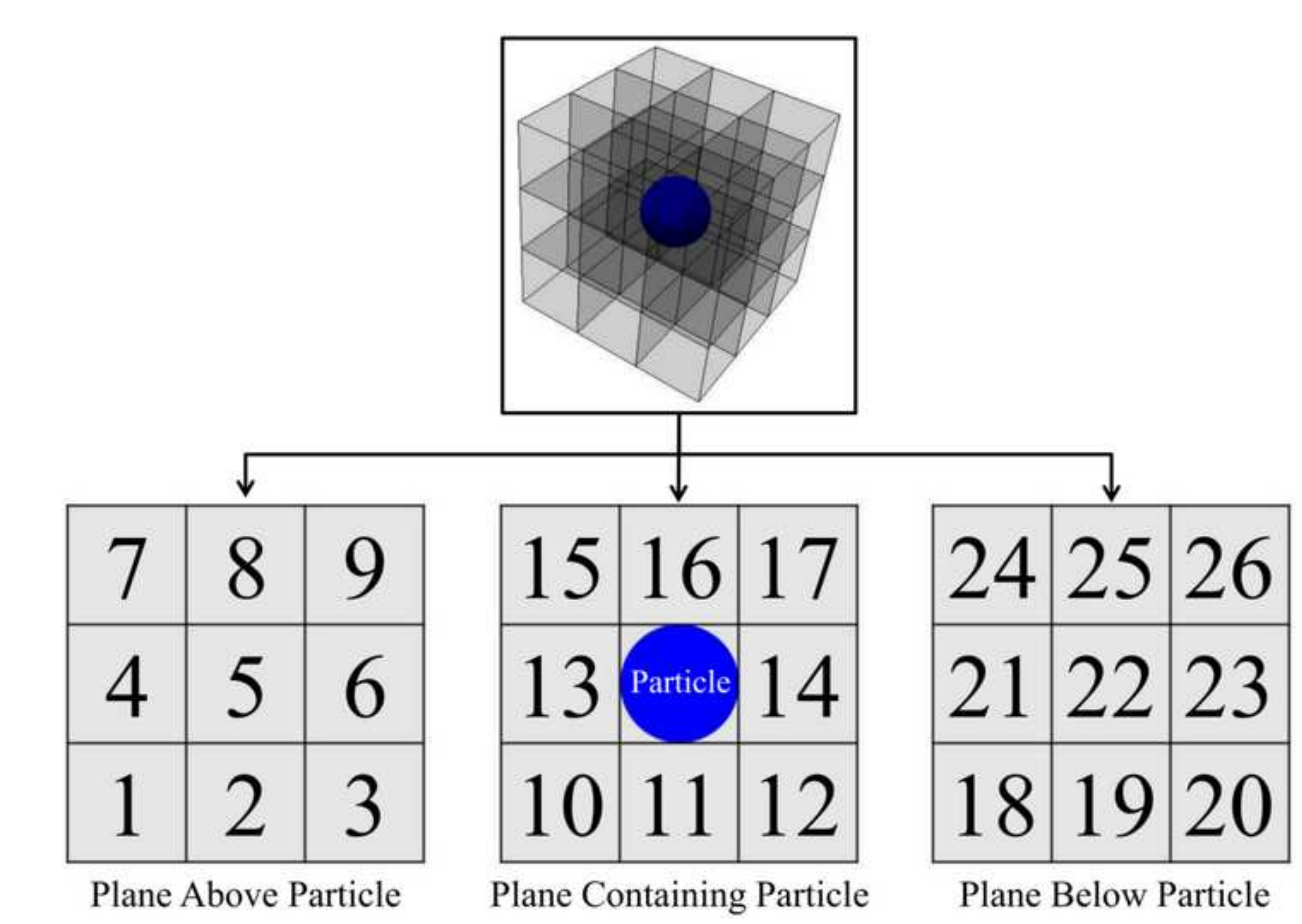

\begin{tabular}{|l|l|l|}
\hline 7 & 0 & 0 \\
\hline 1 & 5 & 6 \\
\hline 1 & 0 & 0 \\
\hline
\end{tabular}

Plane Above Particle
Plane Containing Particle 


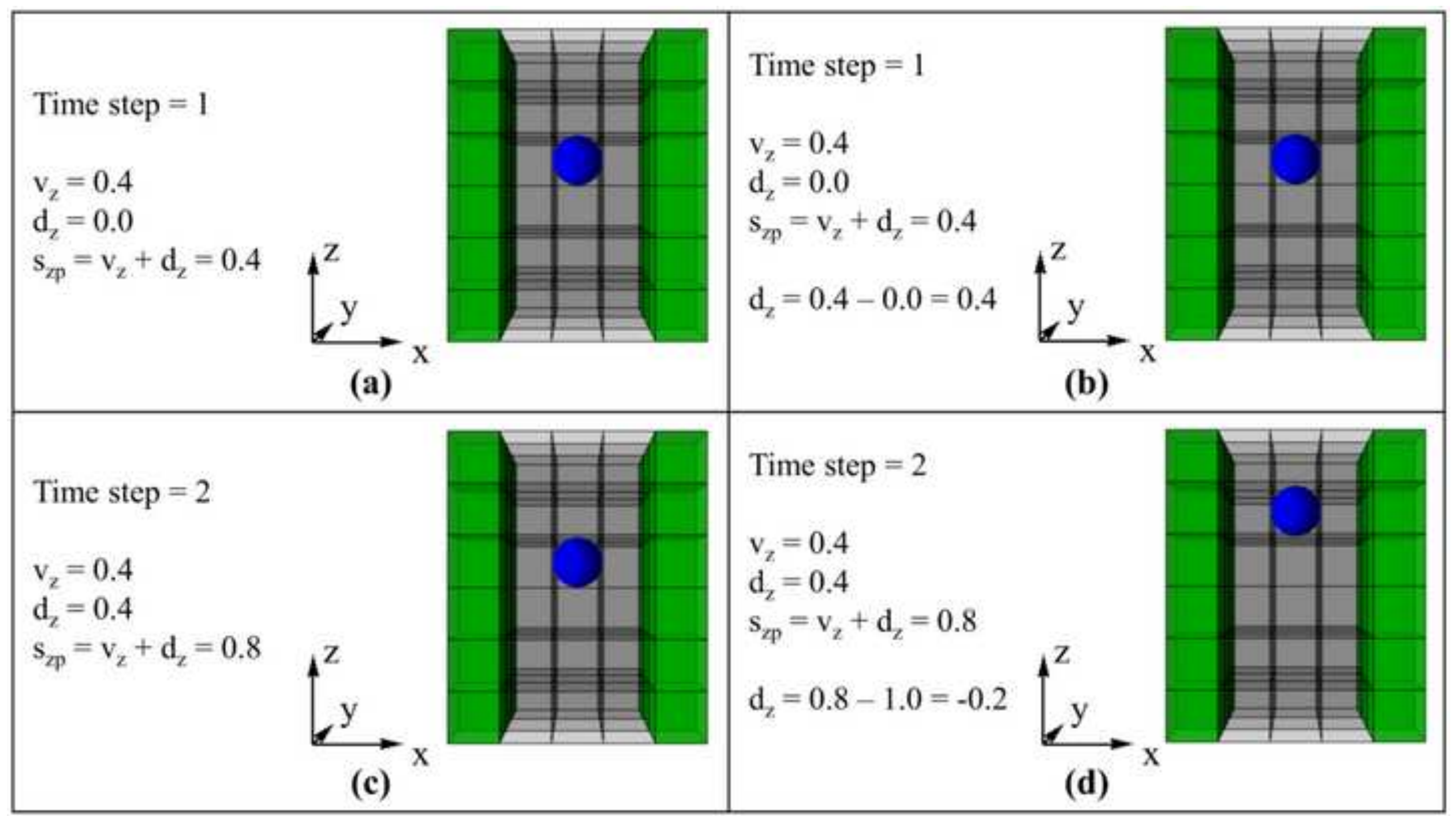


Click here to download high resolution image

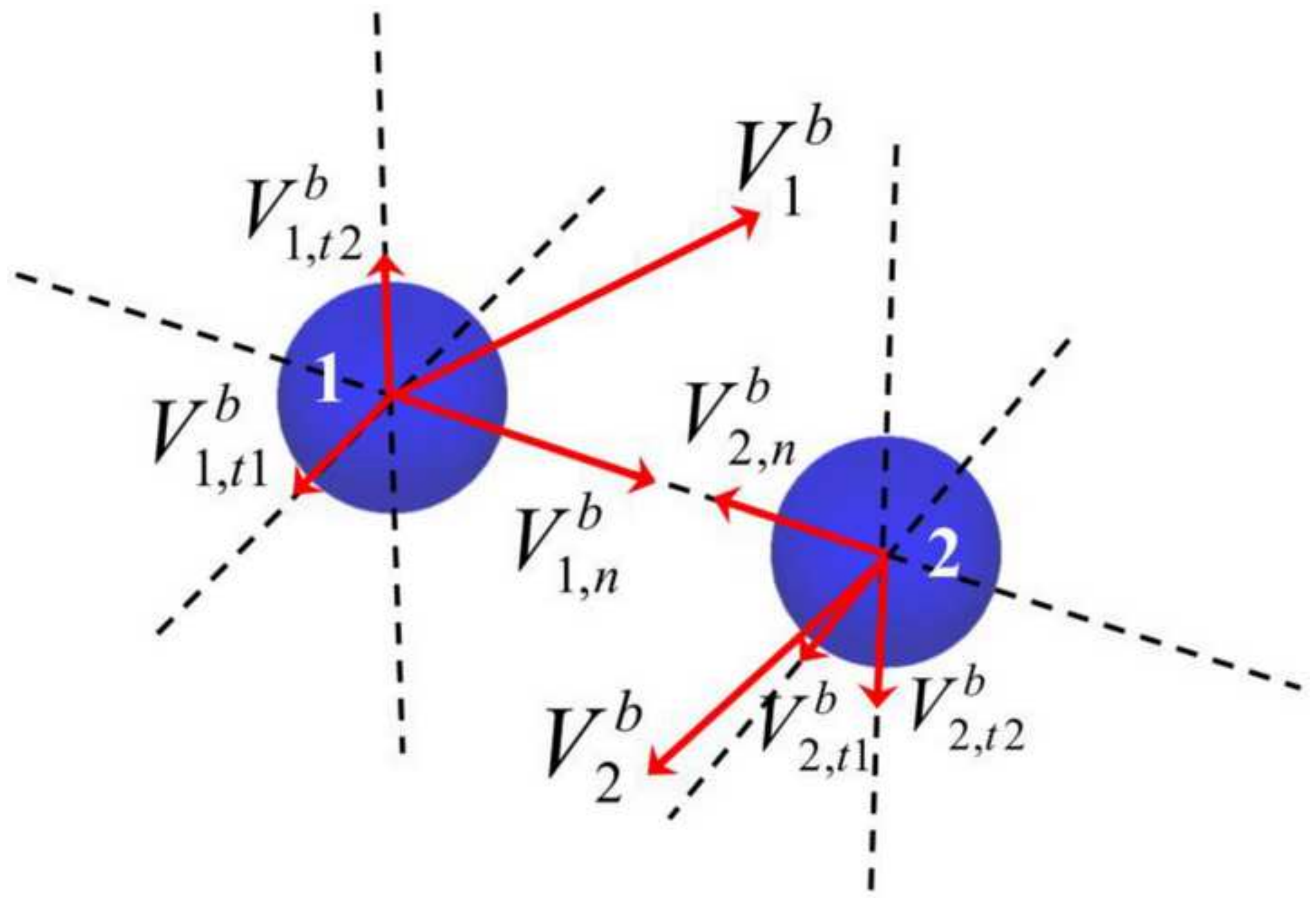




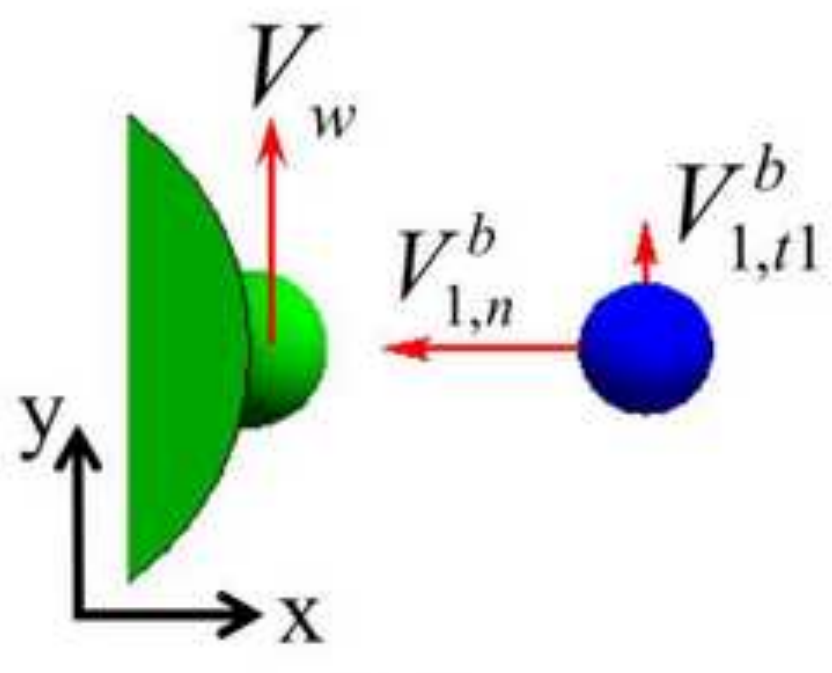

(a)

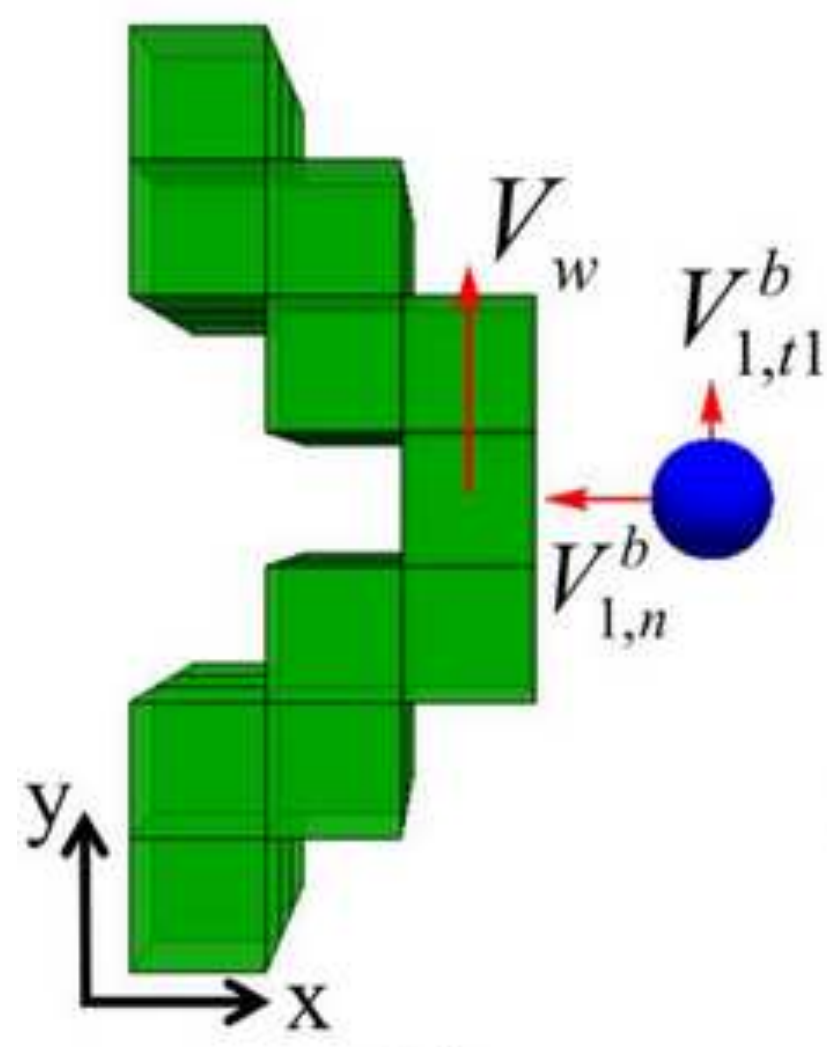

(c)

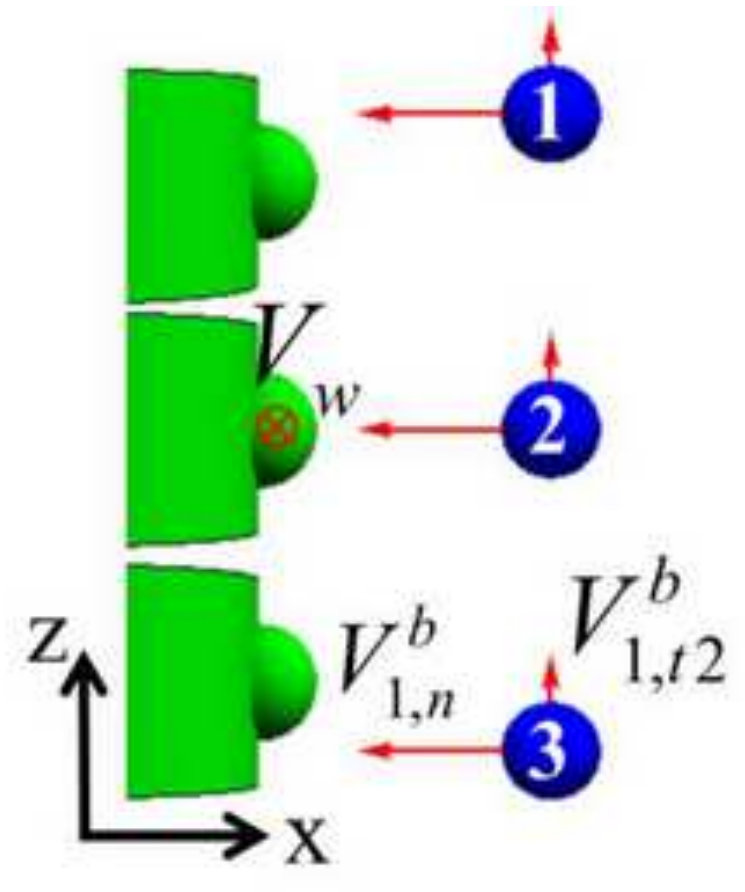

(b)

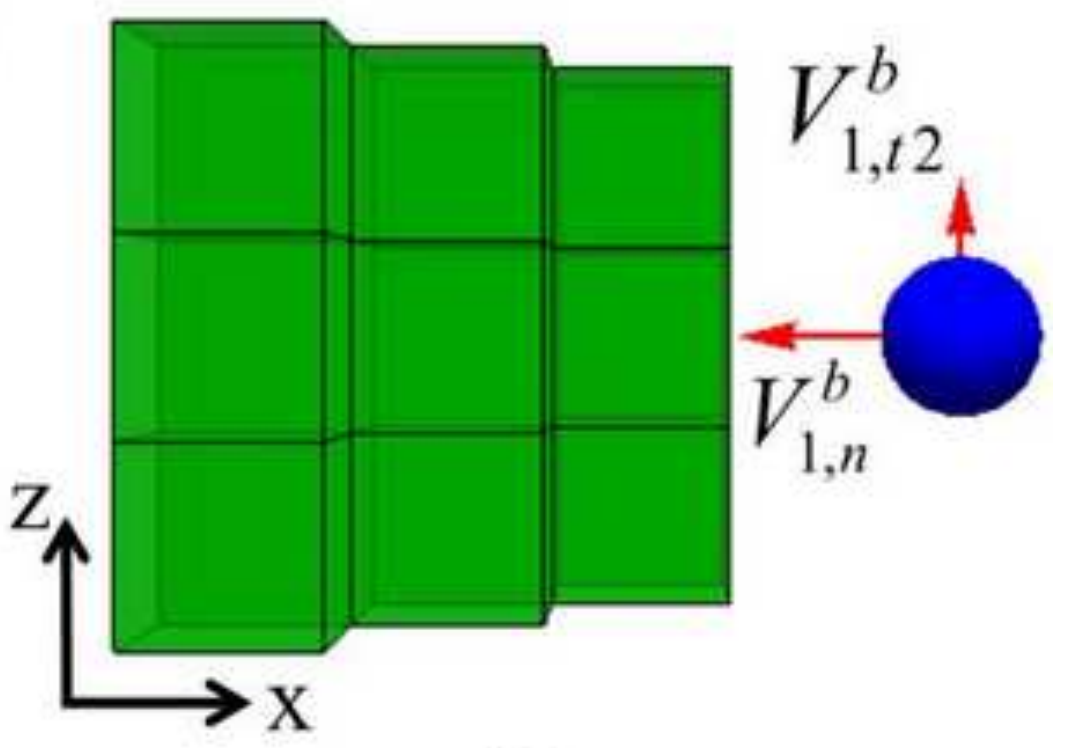

(d) 
Click here to download high resolution image
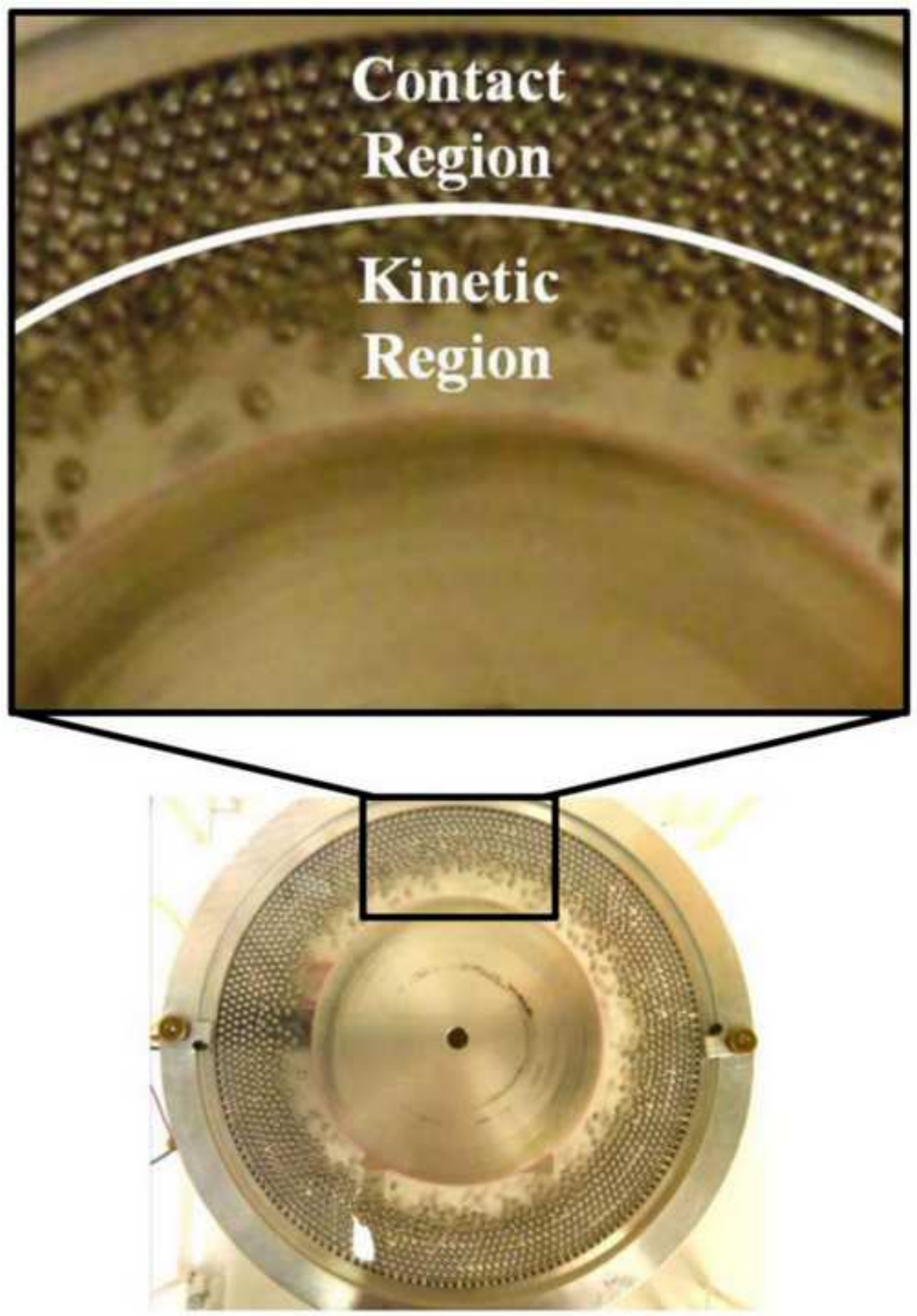


\section{Click here to download high resolution image}

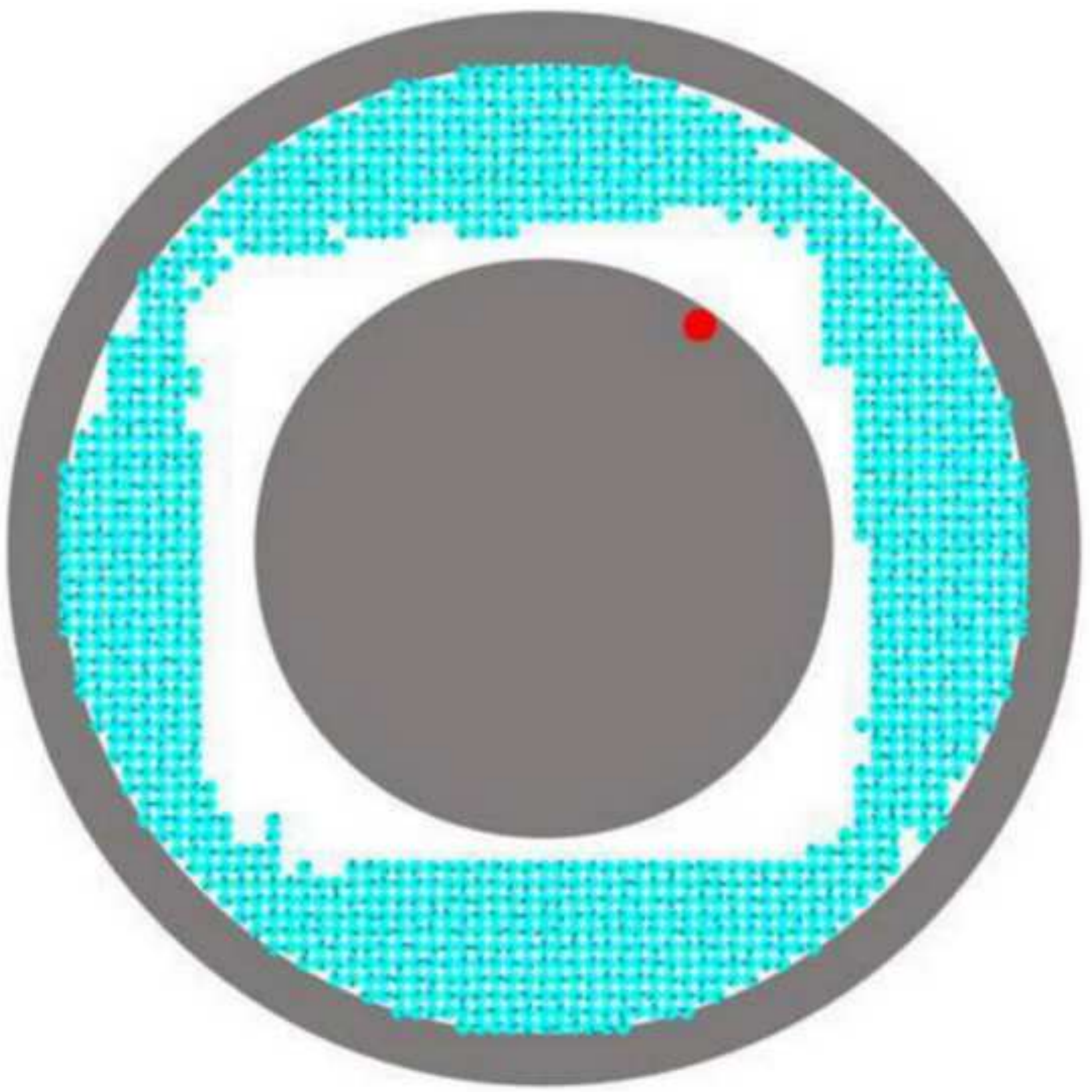


Figure_7

Click here to download high resolution image

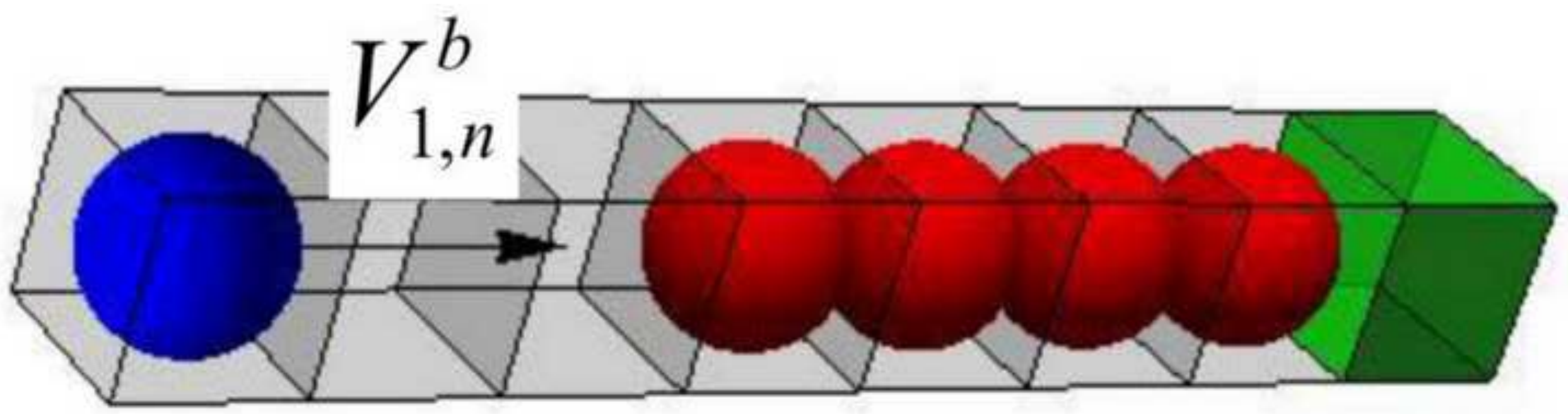



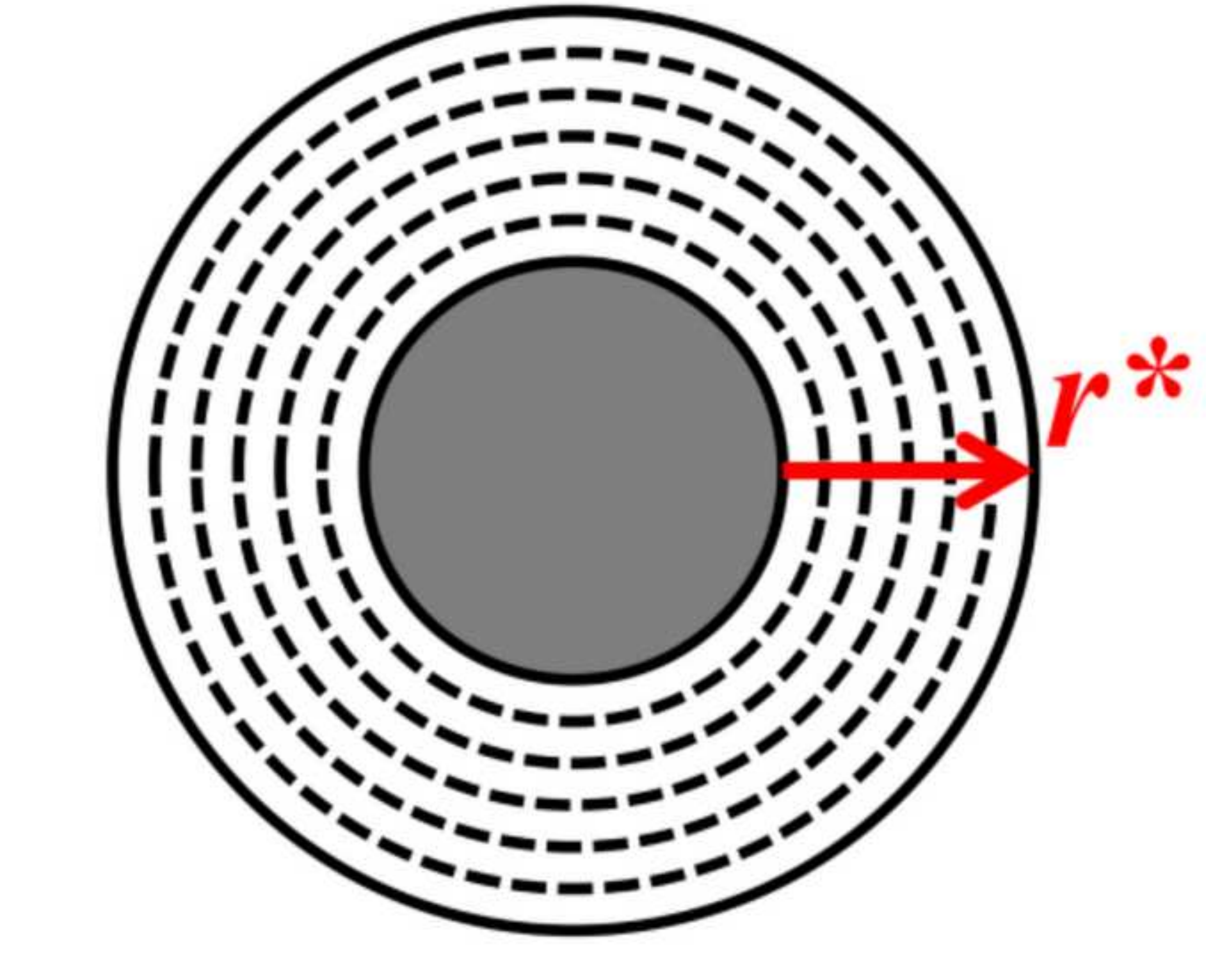


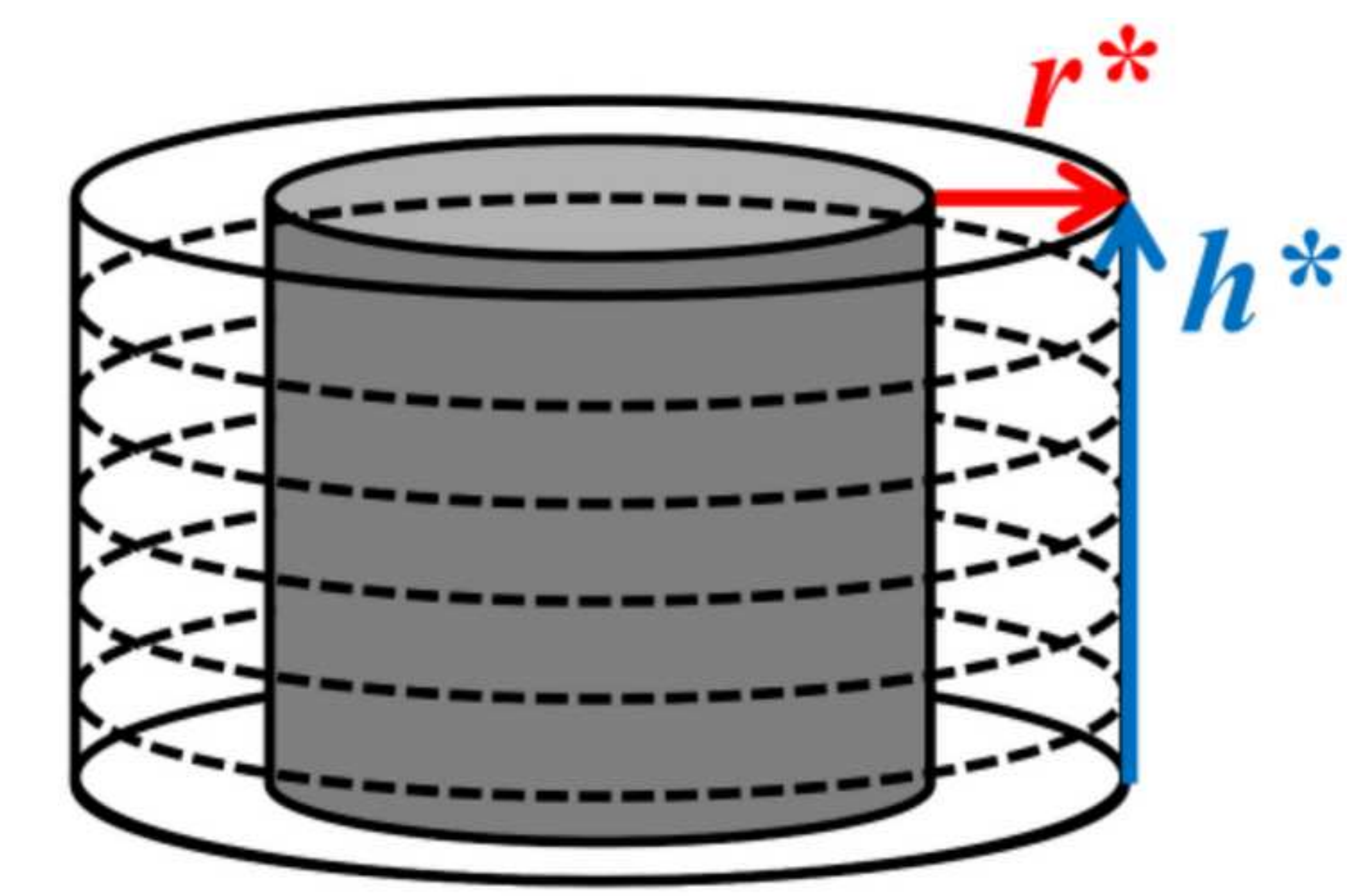

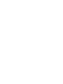

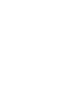
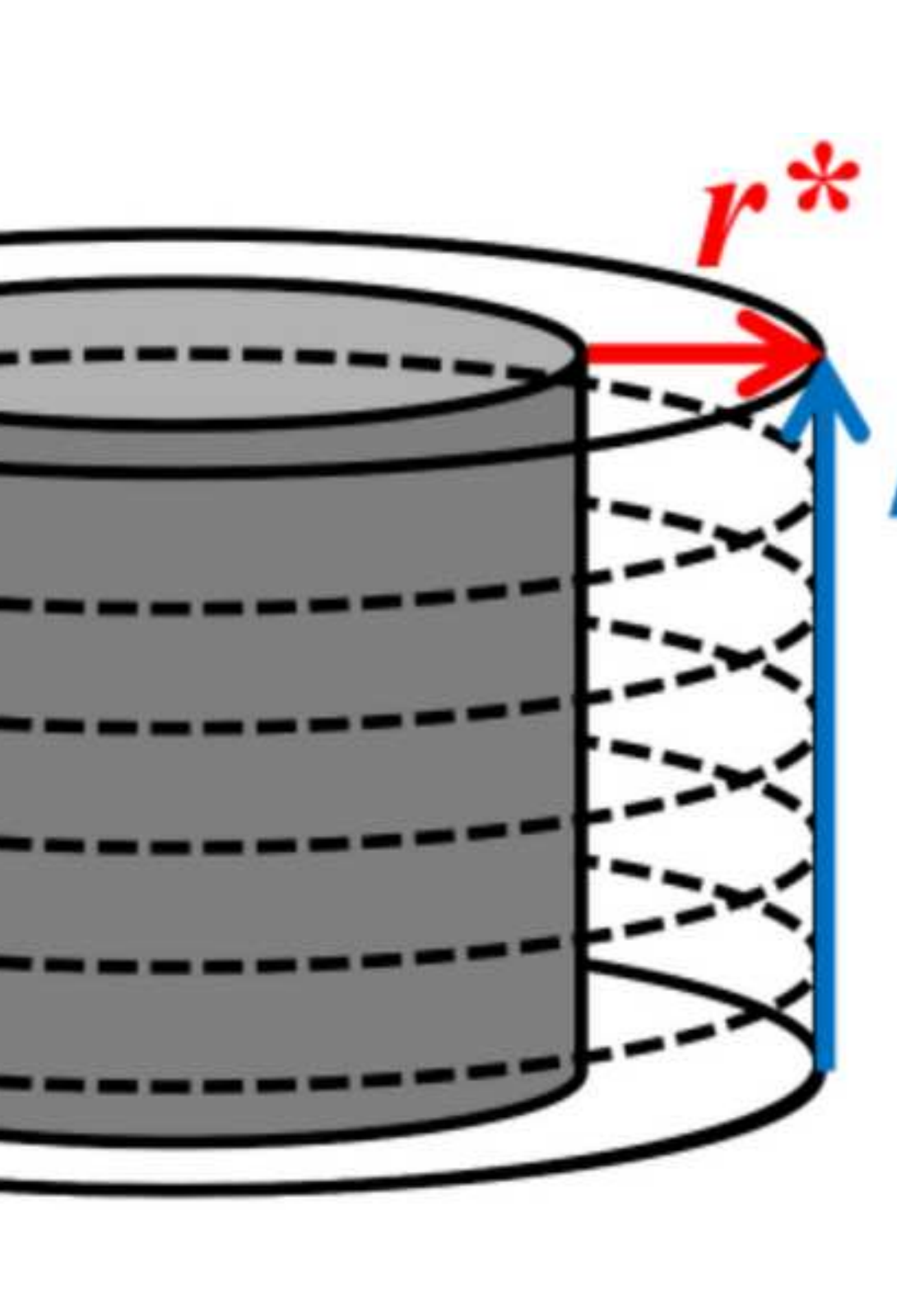

.

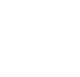
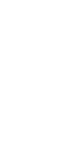

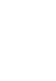

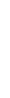


Click here to download high resolution image
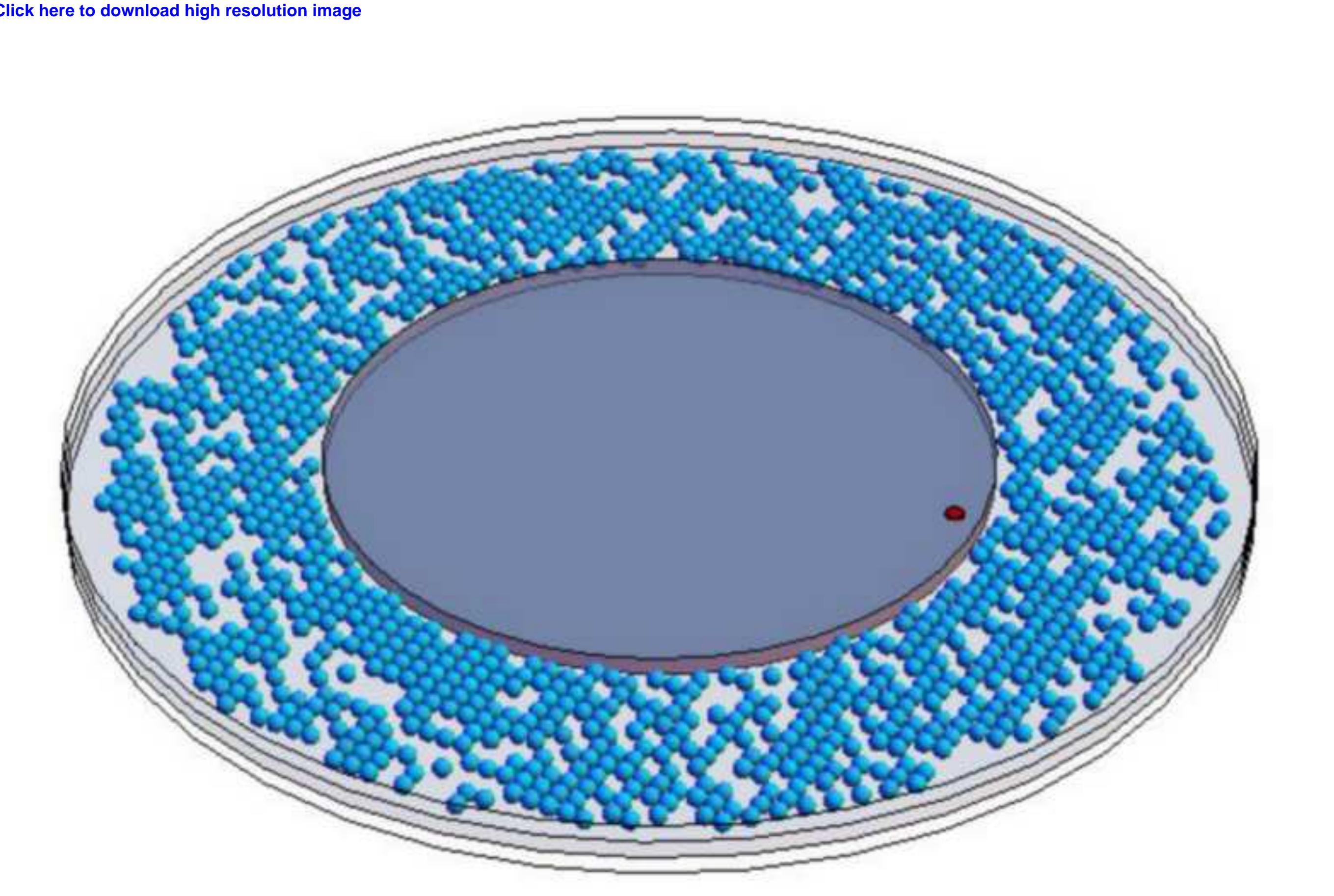

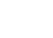


Click here to download high resolution image
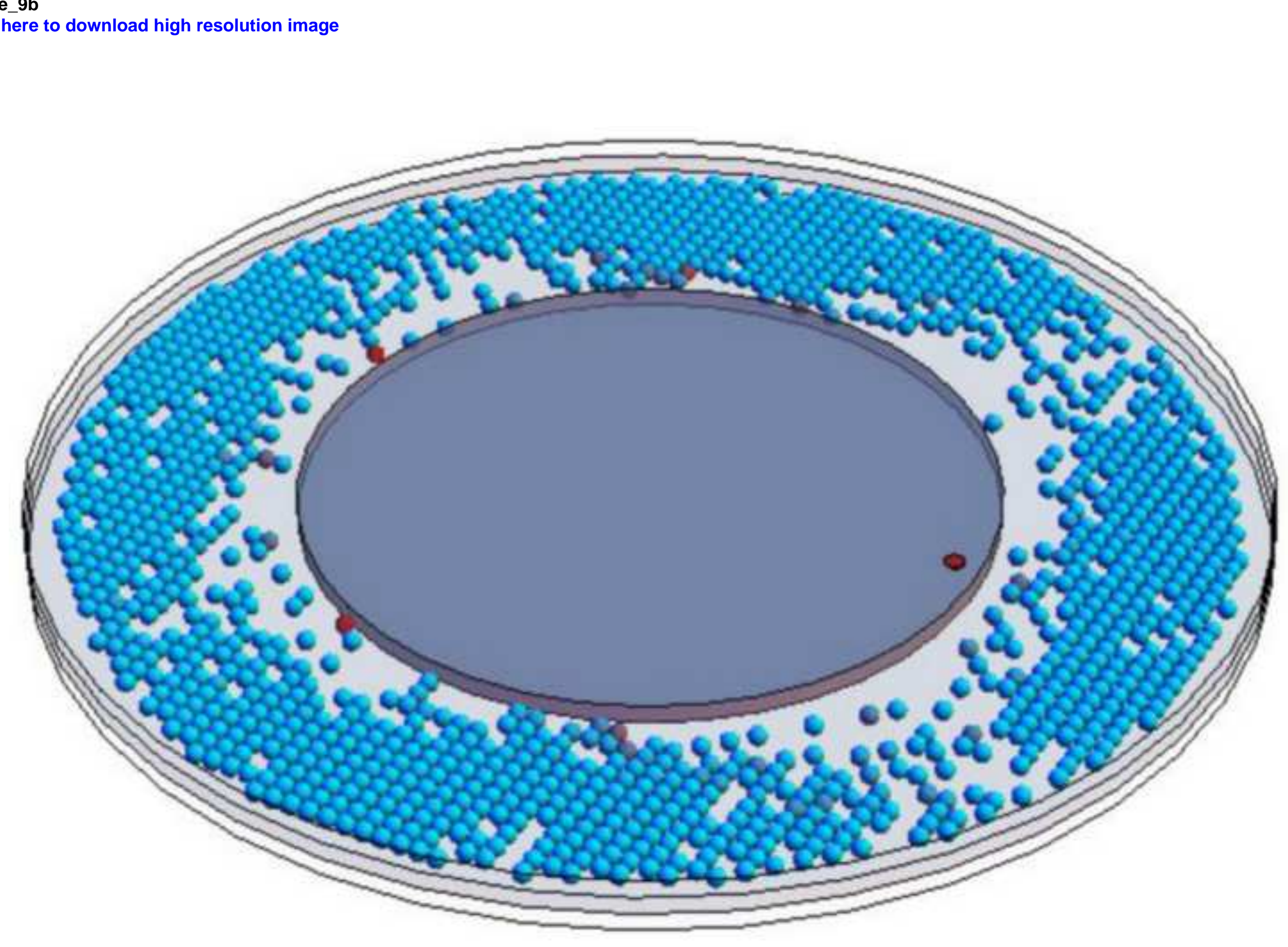

.
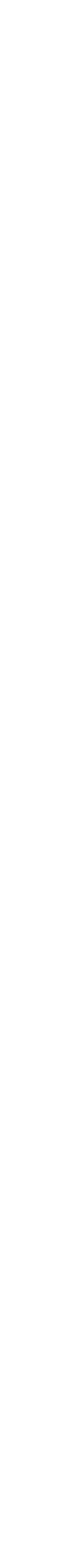
Click here to download high resolution image

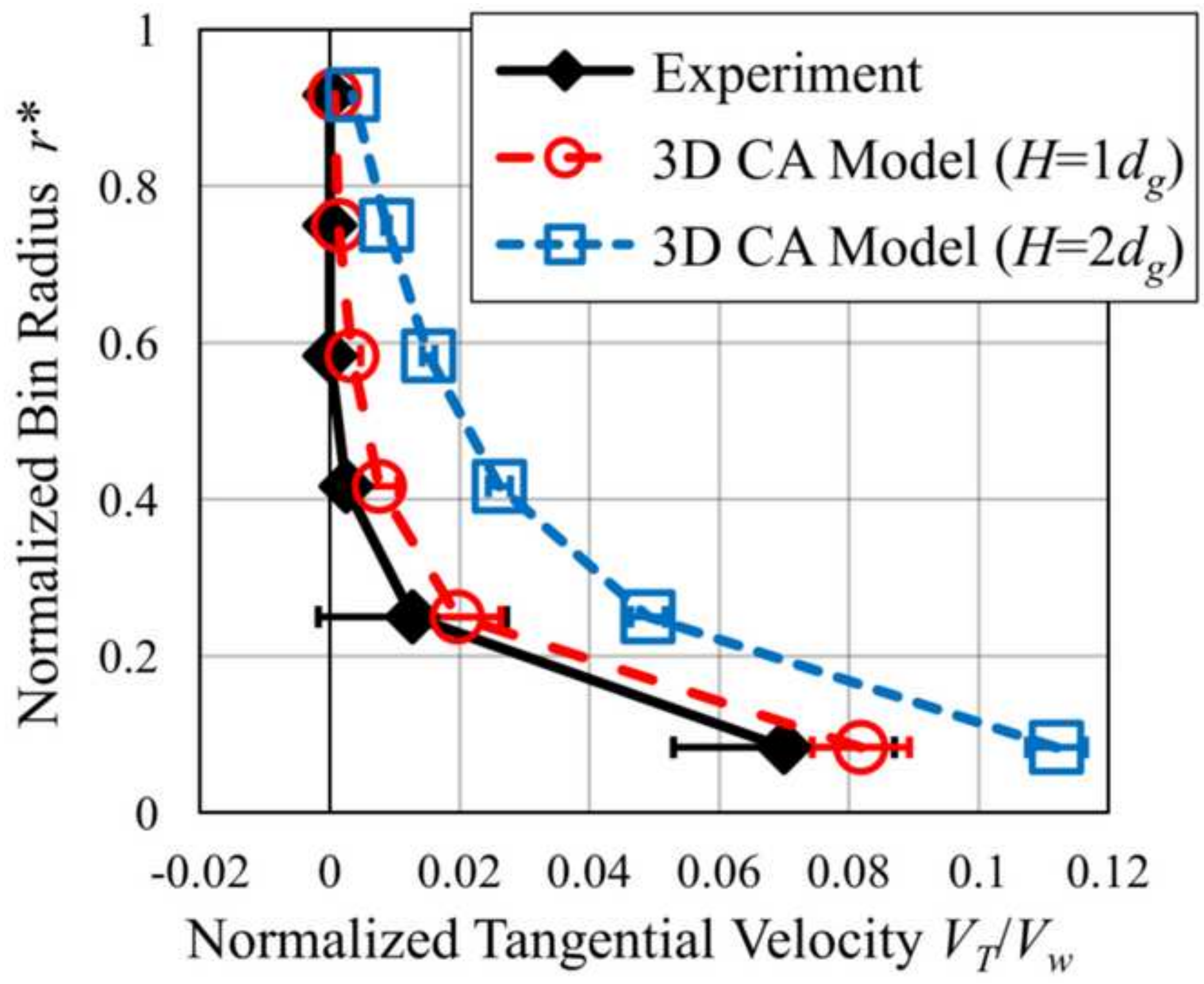




\section{Solid Fraction $v$ (CA Model)}

0

0.2

0.4

0.6

0.8

1

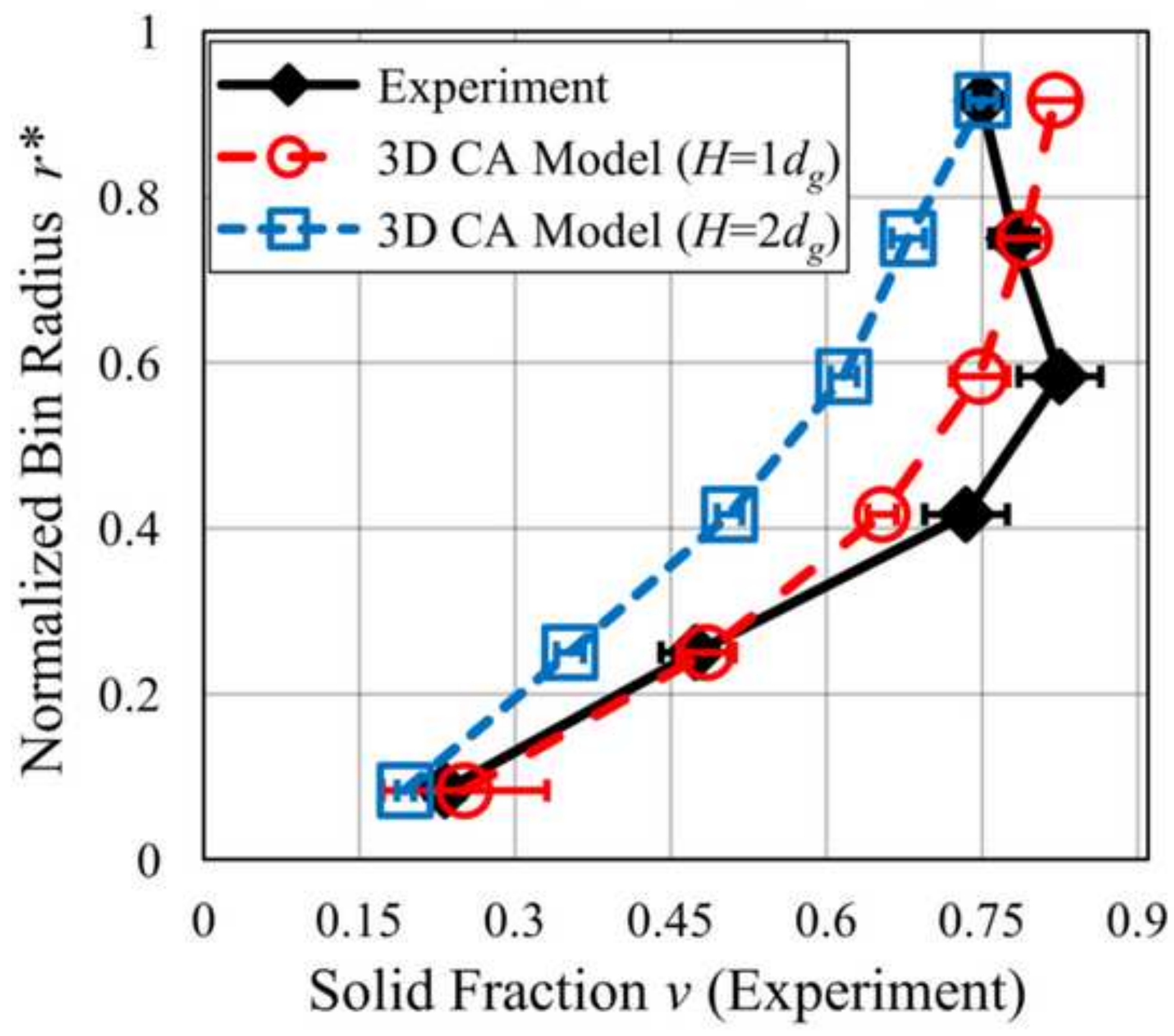




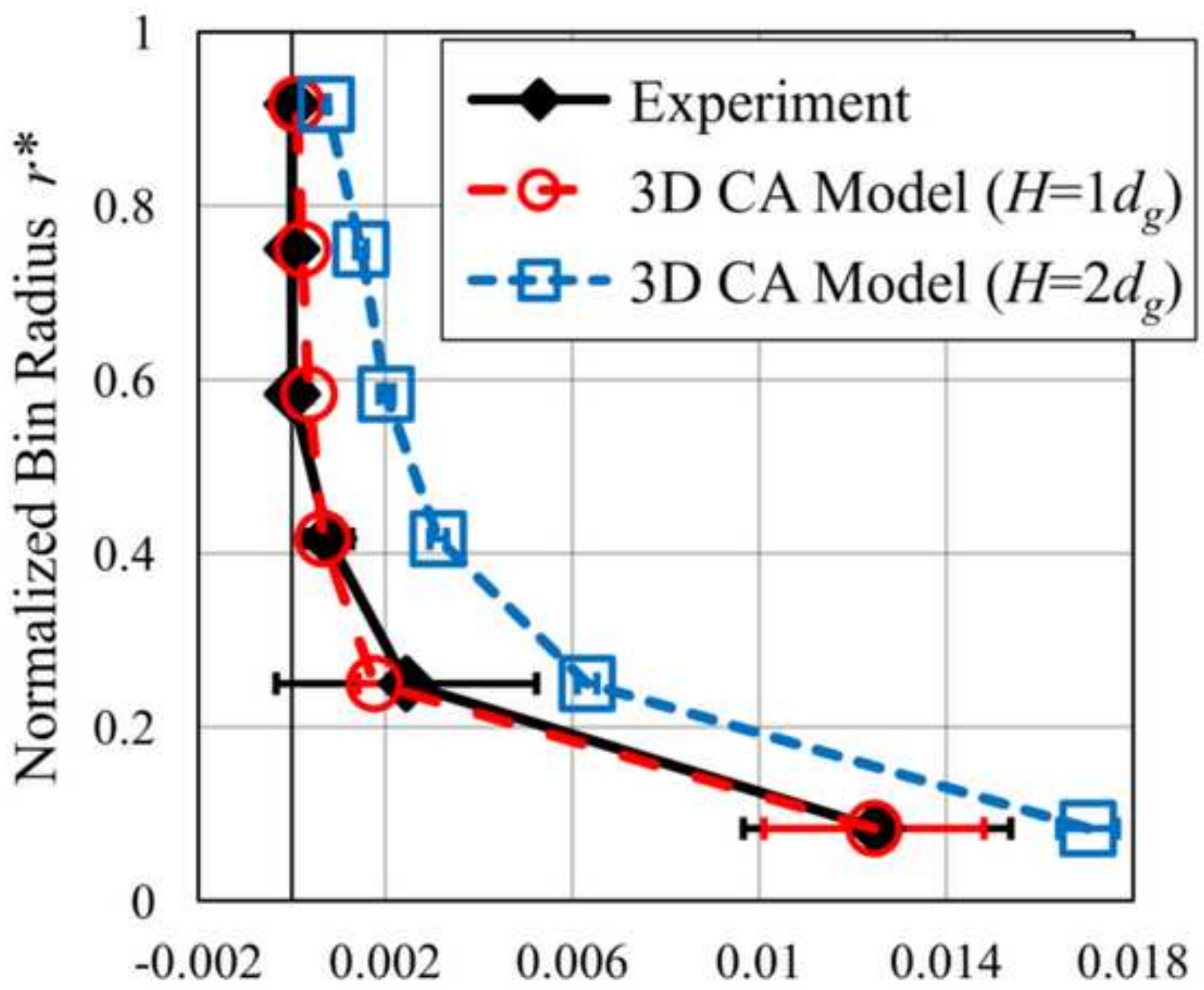

Normalized 2D Granular Temperature $T_{2 D} / V_{w}{ }^{2}$ 
Click here to download high resolution image

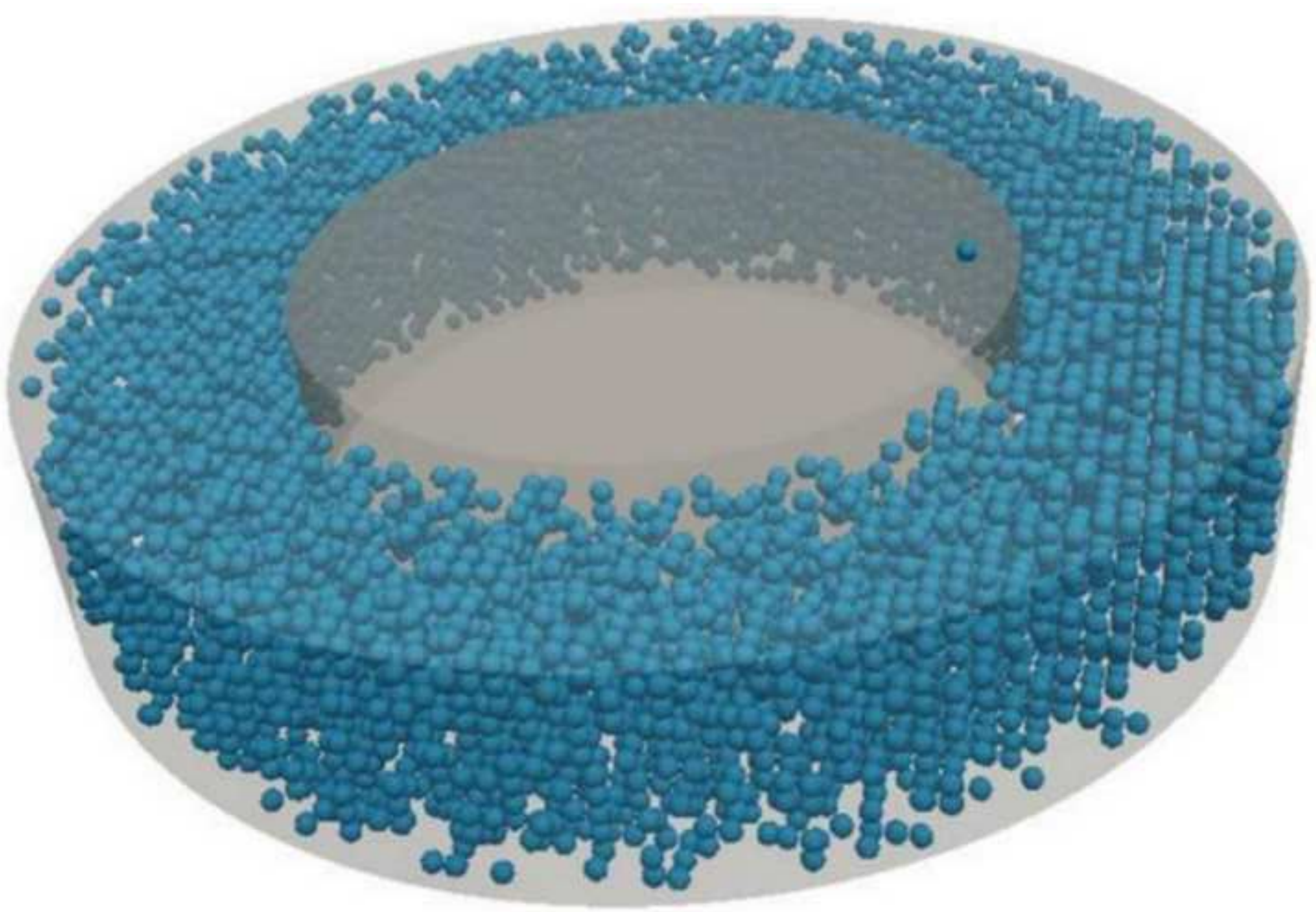


Click here to download high resolution image

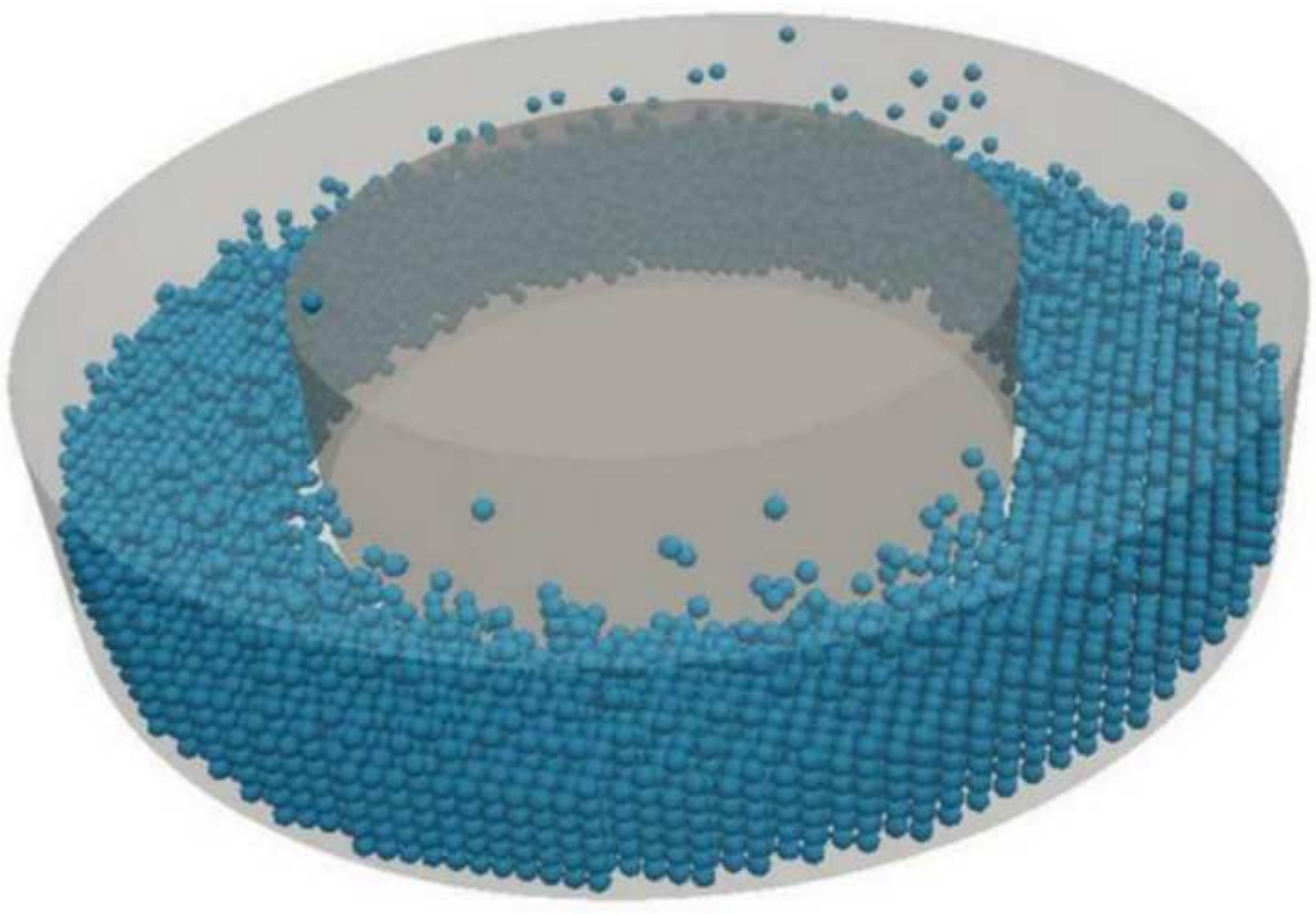




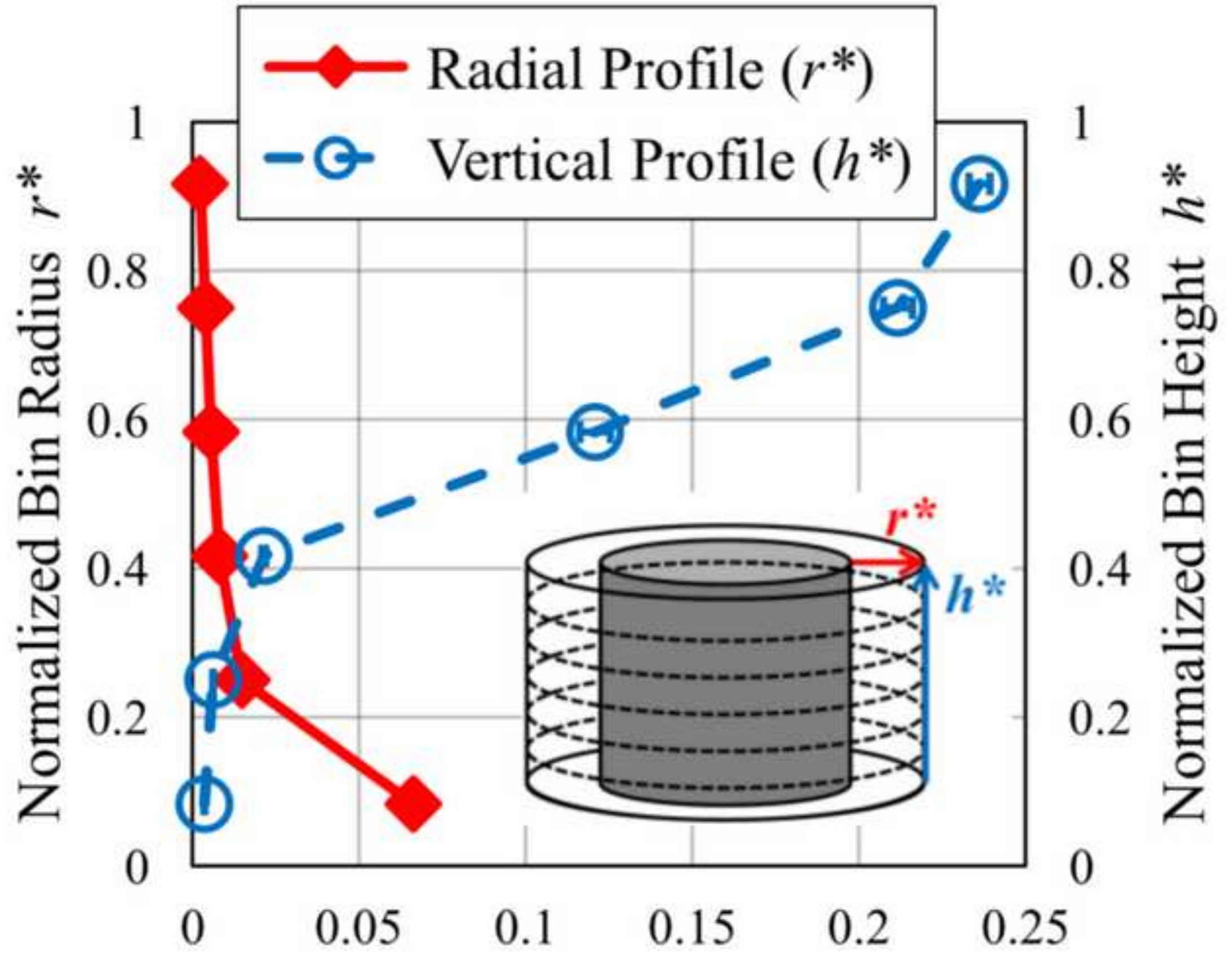

Normalized Tangential Velocity $V_{T} / V_{w}$ 


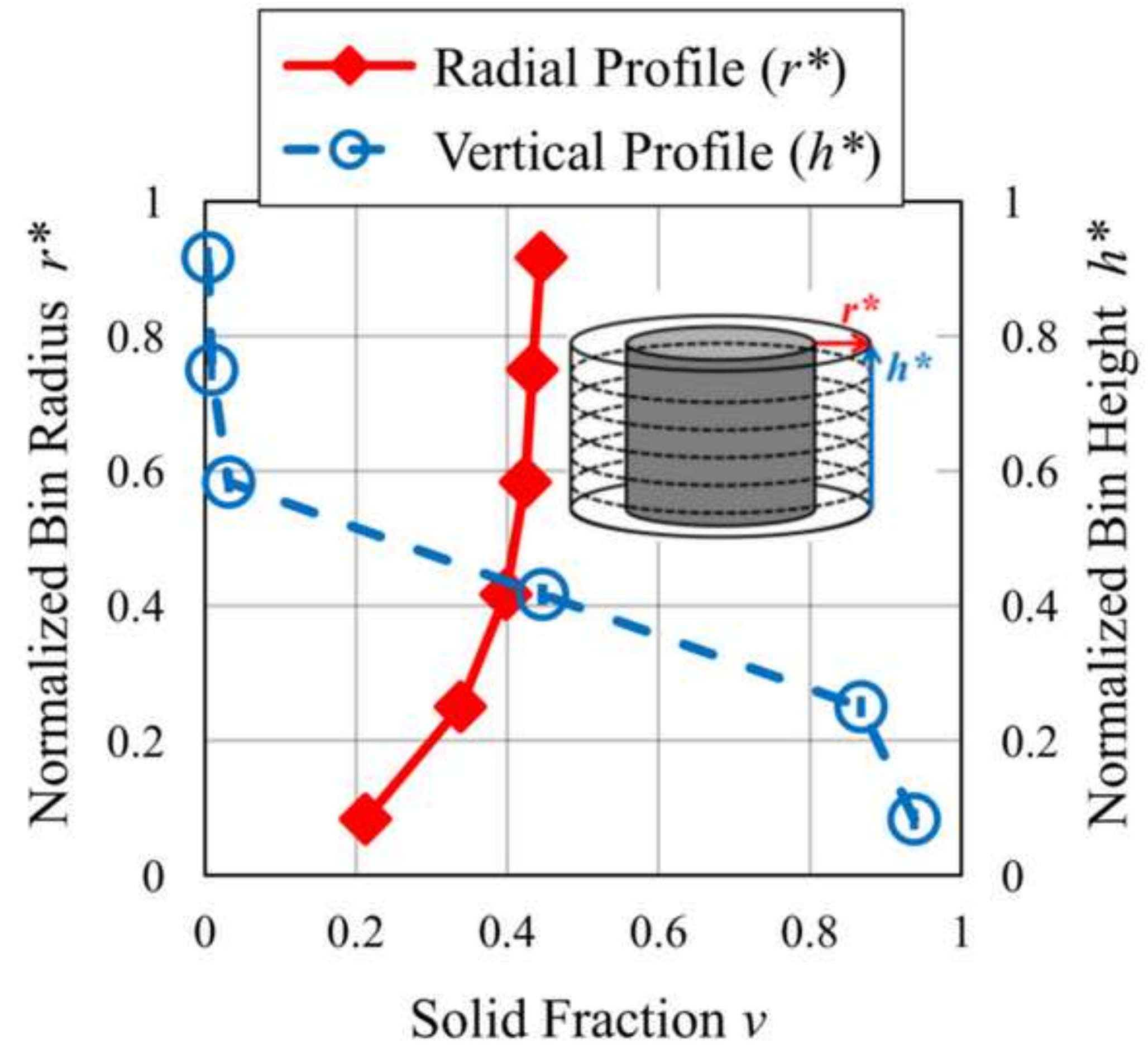




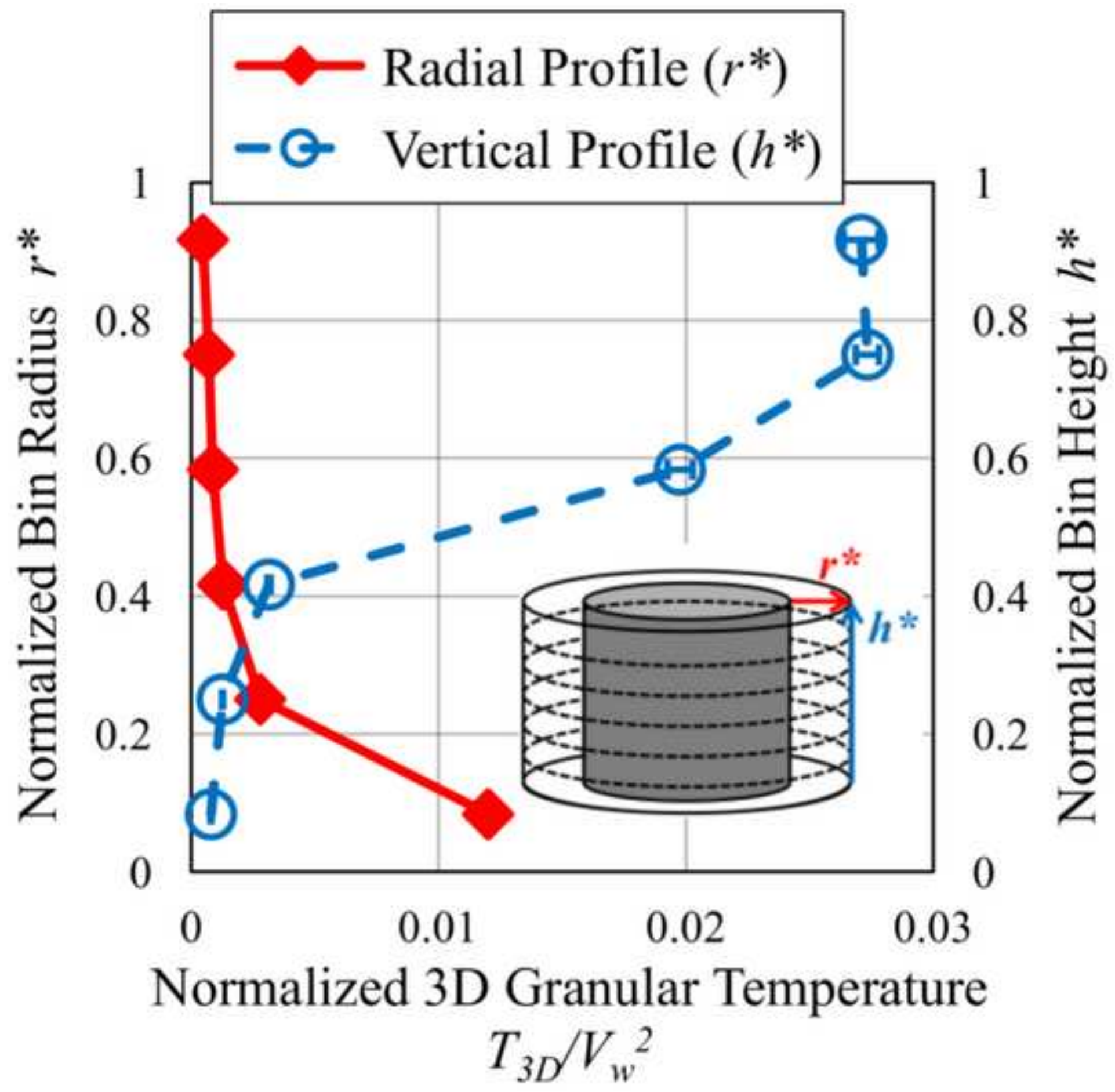




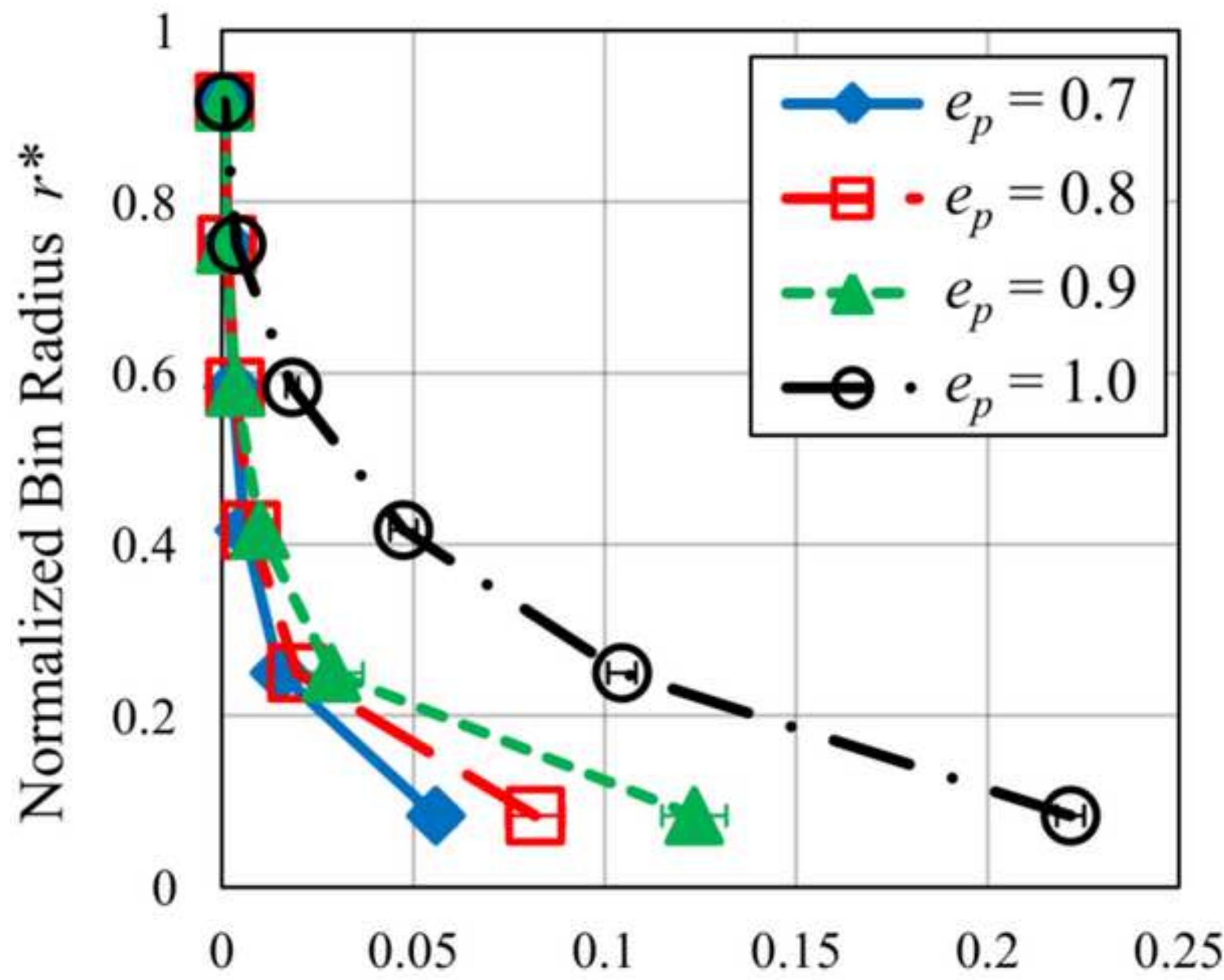

Normalized Tangential Velocity $V_{T} / V_{w}$ 


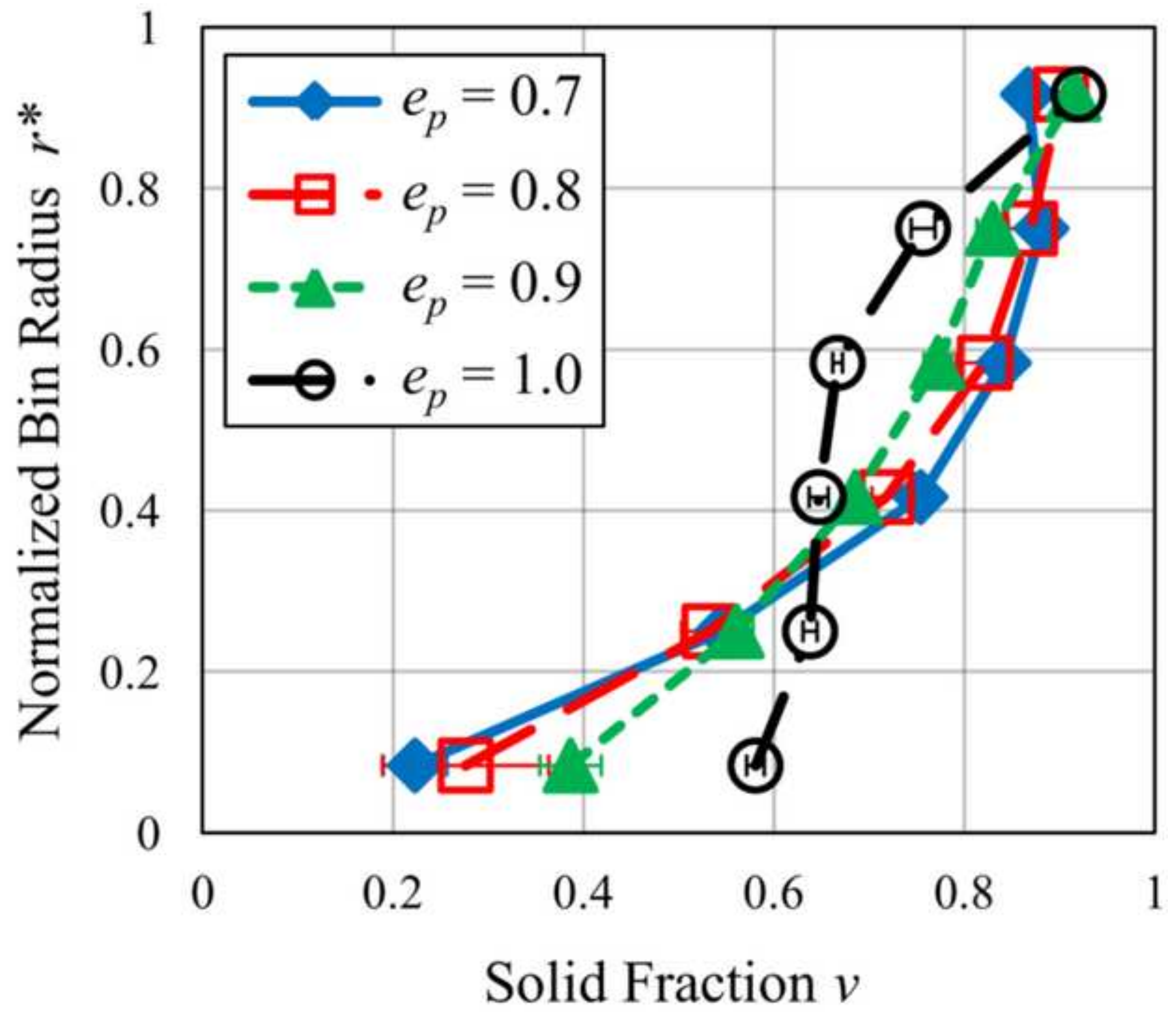



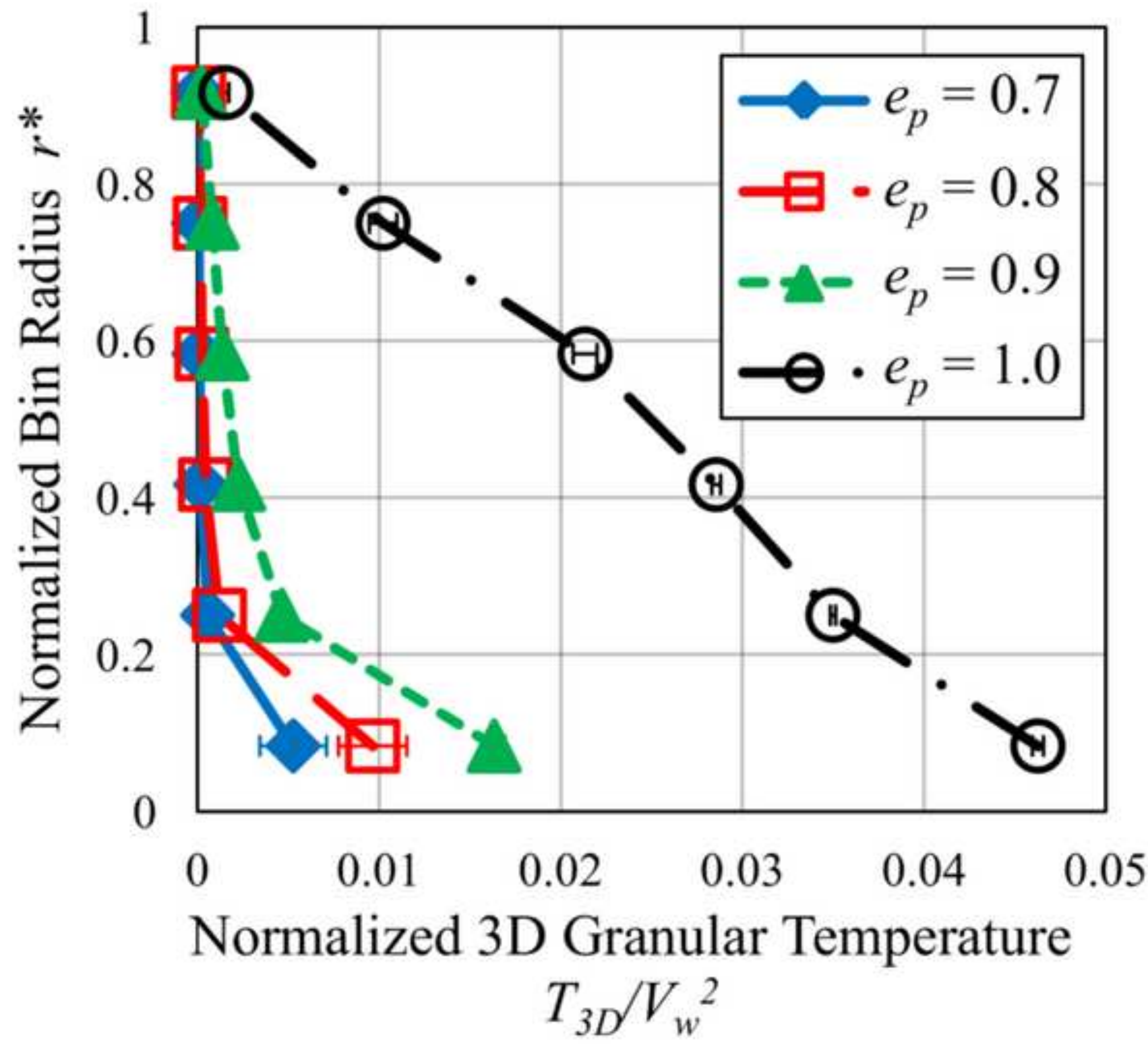


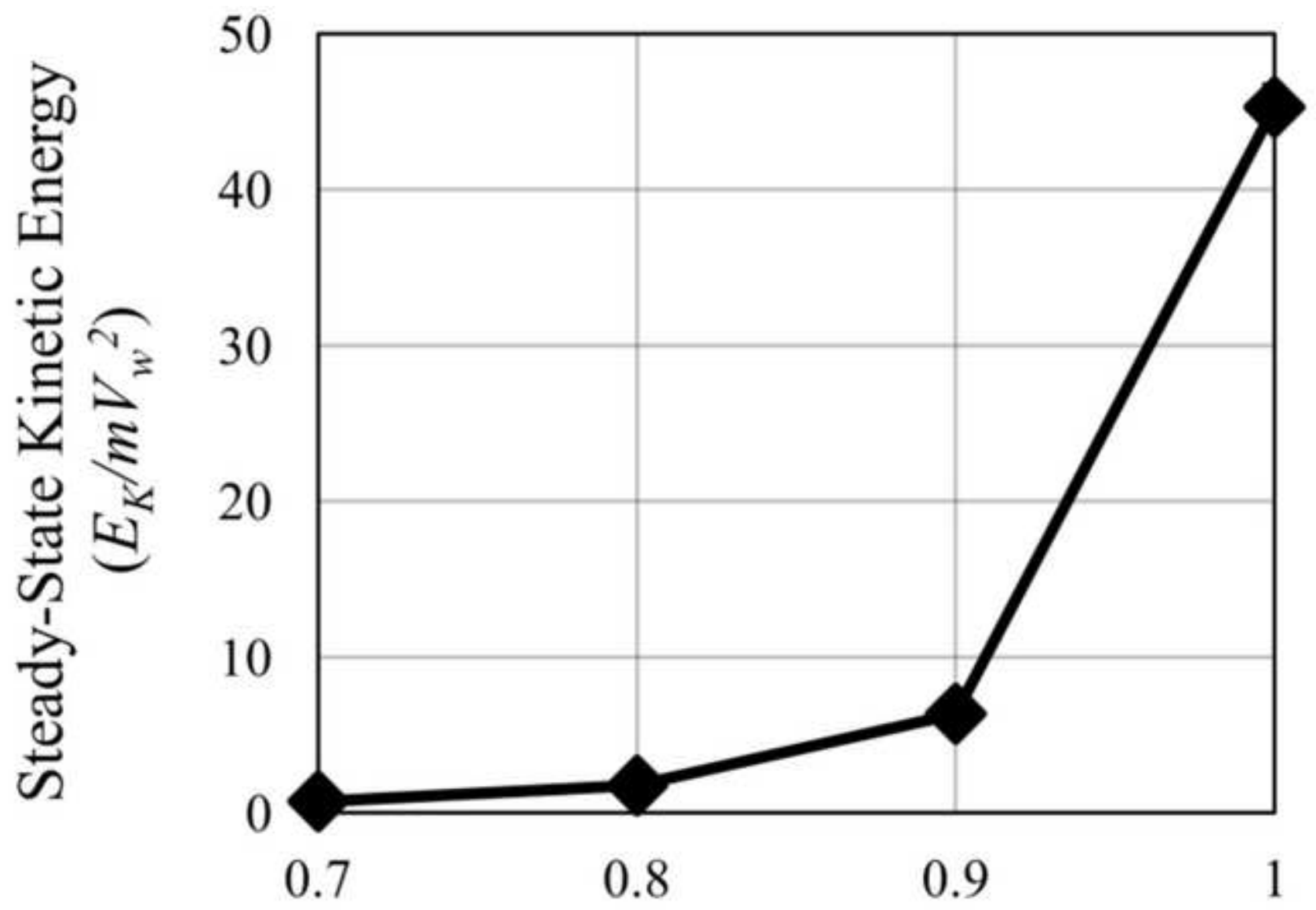

\section{Particle-Particle Coefficient of Restitution $e_{p}$}




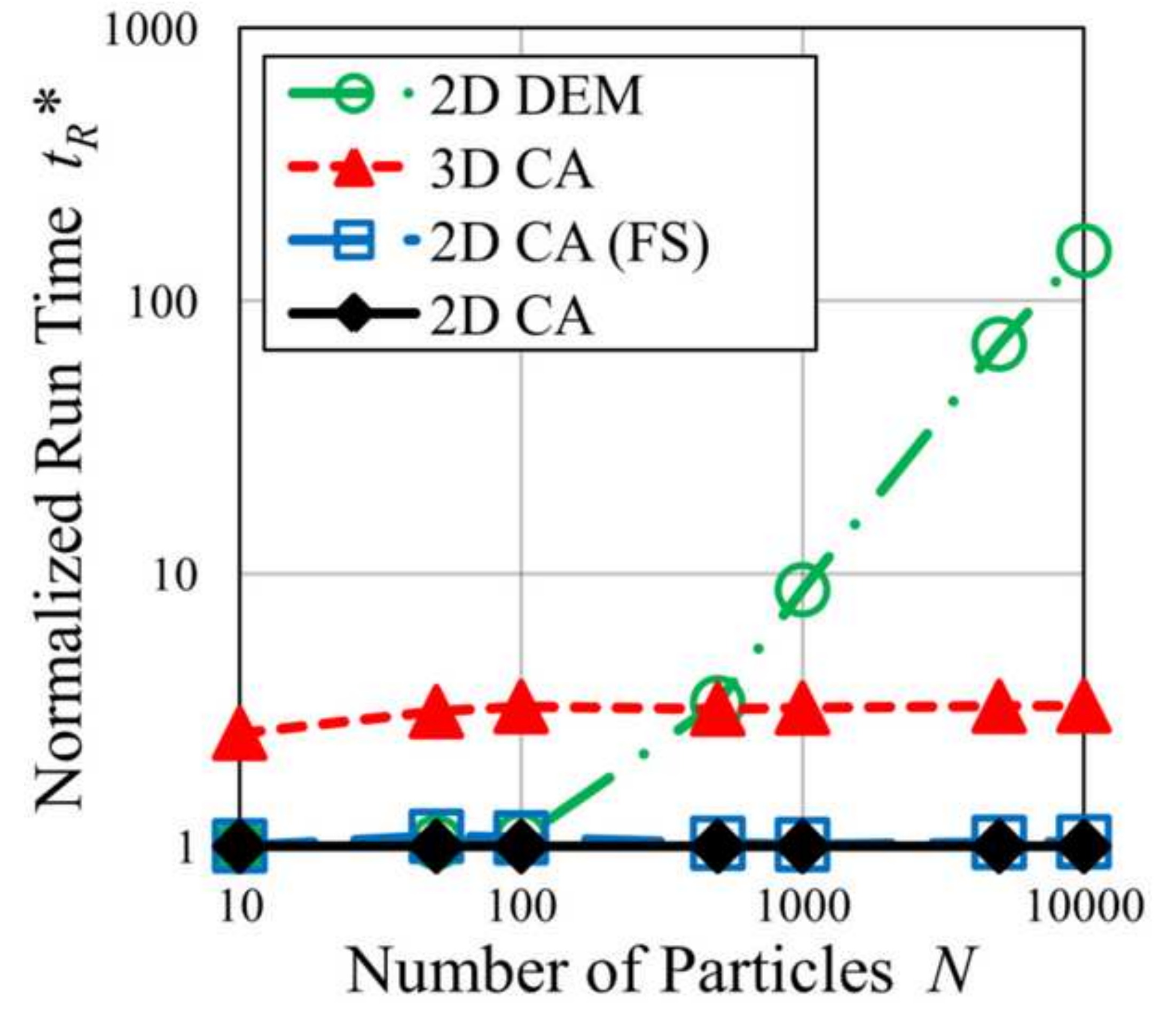

Figure_15
1000

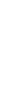




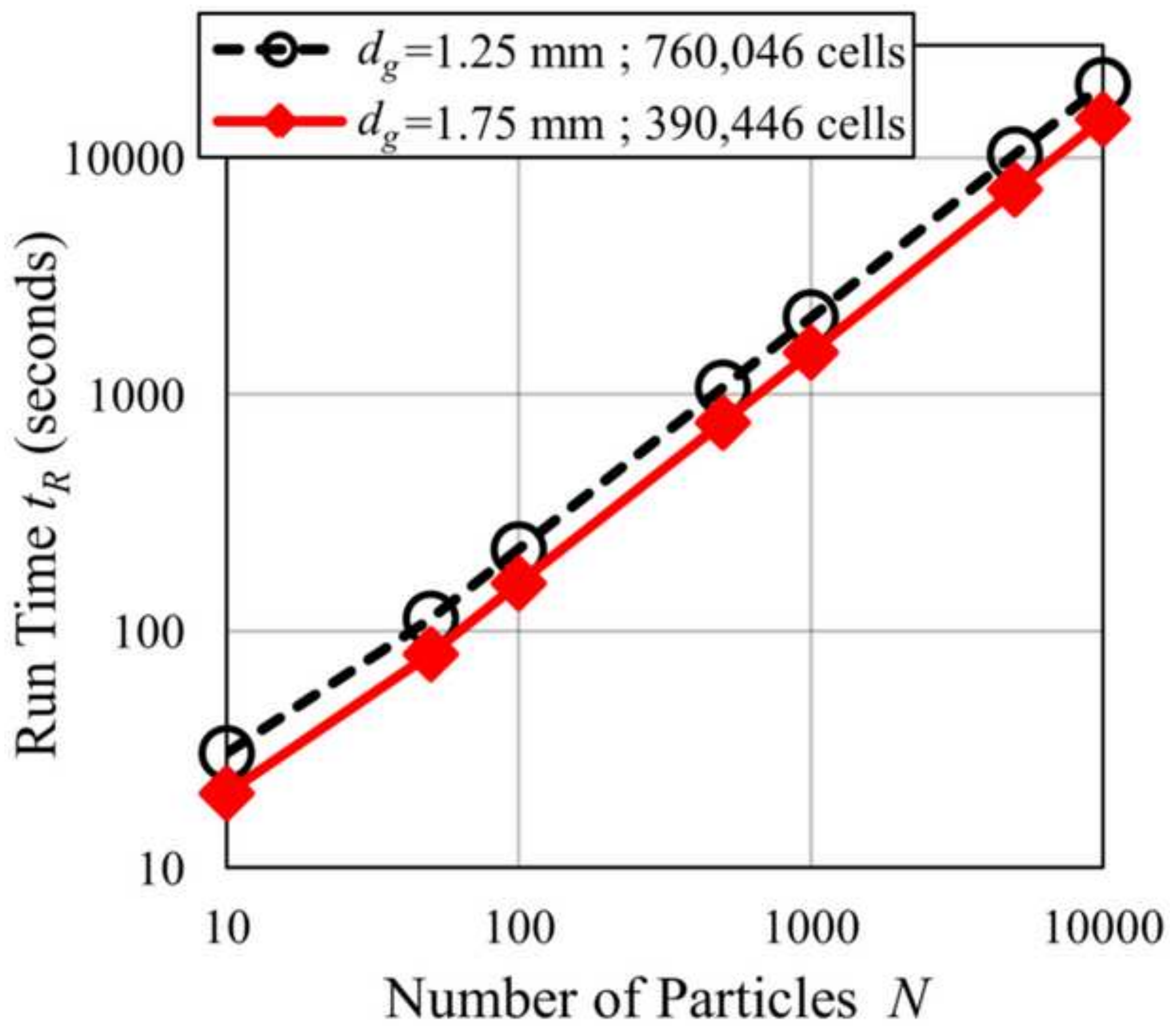




\section{$\underline{\text { Research Highlights }}$}

- A 3D physics-based CA framework for modeling granular flows was developed.

- The 3D CA model provided highly accurate predictions of experimental GSC behavior.

- The model was able to capture highly nonlinear solid fraction behavior.

- The 3D CA model demonstrated a large computational advantage compared to 2D DEM. 
Granular flows continue to be a complex problem in nature and industry where solid particles exhibit solid, liquid, and gaseous behavior in a manner which is often difficult to predict both locally and globally. In solids processing applications such as pharmaceutical production, food processing, and coal processing, the ability to accurately predict particle flows can improve process efficiency and product effectiveness. The "gold standard" for the modeling and prediction of granular flows is the discrete element method (DEM), which provides a rigorous physical treatment of particle interactions. One possible supplement to DEM-based analysis is lattice-based cellular automata (CA). CA provides a platform for obtaining fast predictions by pairing rule-based mathematics with high-fidelity physics to rapidly model physical processes, such as granular flows. The present work develops a novel three-dimensional (3D) physics-based CA framework for modeling granular shear flows. This 3D CA model extends the framework's modeling capabilities beyond the granular kinetic regime, includes treatments for force chains, and provides reasonably high accuracy in terms of predicting fundamental granular flow experiments. The predictive capabilities and quantitative accuracy of this CA model have been tested and validated against experimental shear flow results from an annular shear cell. Results from this validation work suggest that the model accurately predicts local flow properties. A parametric study varying the particle-particle coefficient of restitution demonstrated the ability of the 3D CA model to capture nonlinear trends in the local solid fraction profiles. A time study was also performed to highlight the computational efficiency of the 3D CA framework developed in this work. Results of this study showed that even at relatively low particle counts $\left(10^{4}\right)$, the 3D CA model provides for considerable computational savings, with run times of two orders of magnitude less than a 2D DEM model. 


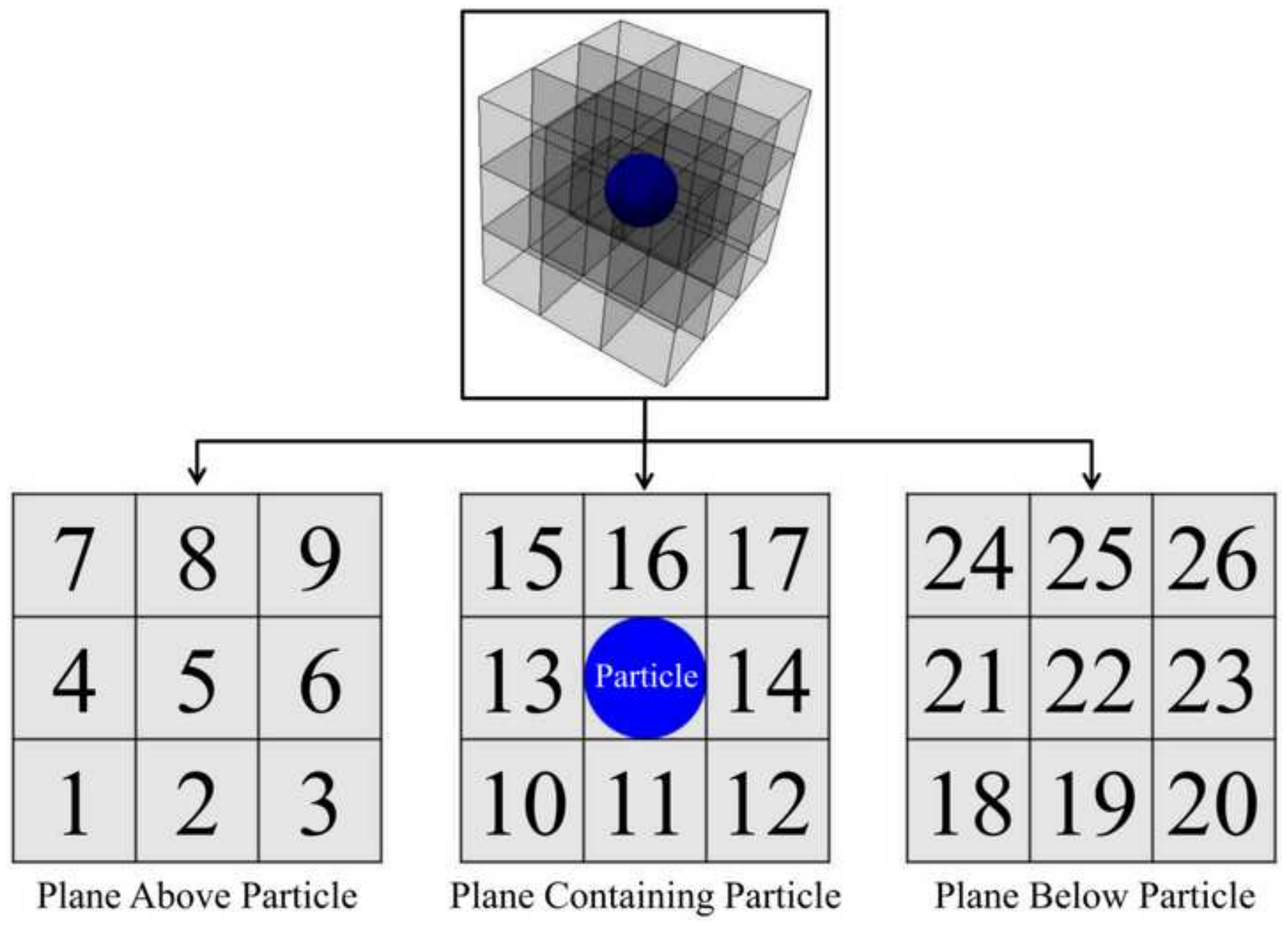


The "gold standard" for modeling granular flows is the discrete element method (DEM). One possible supplement to DEM is lattice-based cellular automata (CA), which can provide fast predictions using rule-based mathematics and high-fidelity physics. This work develops a novel three-dimensional (3D) physics-based CA framework for modeling granular shear flows. 